\title{
Avoidance of singularities in spherically symmetric charged dust
}

\author{
Andrzej Krasiński and Krzysztof Bolejko* \\ N. Copernicus Astronomical Center, Polish Academy of Sciences \\ Bartycka 18, 00716 Warszawa, Poland \\ email: akr@camk.edu.pl
}

\begin{abstract}
In spherically symmetric charged dust, just like in neutral dust, two kinds of singularity may be present: the Big Bang/Crunch (BB/BC) singularity, and shell crossings. Quite unlike in neutral dust, the $\mathrm{BB} / \mathrm{BC}$ singularity may be avoided. When the charge density $\rho_{e}$ and the mass-energy density $\epsilon$ obey $\left|\rho_{e}\right|<D_{e} \stackrel{\text { def }}{=} \sqrt{G} \epsilon / c^{2}$, the conditions that allow the model to avoid the $\mathrm{BB} / \mathrm{BC}$ singularity necessarily lead to shell crossings. However, when $\left|\rho_{e}\right| \rightarrow D_{e}$ at the center of symmetry while $\left|\rho_{e}\right|<D_{e}$ elsewhere, both kinds of singularity may be avoided for a sufficiently long period that a body of charged dust may go through the tunnel between the singularities in the maximally extended Reissner - Nordström spacetime, to emerge into another asymptotically flat region. An explicit example of such a configuration is presented and discussed. It does not contradict any astrophysical constraints.
\end{abstract}

\section{Spherically symmetric dust in electromagnetic field}

For the spherically symmetric spacetimes in the comoving coordinates, the metric can be put in the form

$$
\mathrm{d} s^{2}=\mathrm{e}^{C(t, r)} \mathrm{d} t^{2}-\mathrm{e}^{A(t, r)} \mathrm{d} r^{2}-R^{2}(t, r)\left[\mathrm{d} \vartheta^{2}+\sin ^{2}(\vartheta) \mathrm{d} \varphi^{2}\right]
$$

The case $R,_{r}=0$ requires separate consideration. In this case, a solution of the Einstein equations for electrically neutral dust was found by Datt [1] and then derived and interpreted by Ruban [2, 3]; the corresponding Einstein-Maxwell equations were systematically derived and solved by Ruban [4, 5, 6].

We will discuss the solutions of the Einstein-Maxwell equations in the generic case $R,,_{r} \neq 0$. Most of the material of Sections 2 to 7 has been known for a long time, but a few points were overlooked by some of the earlier authors. That material is reviewed here in order to introduce the framework and notation, and to fill in the few little gaps. New results are reported in Sections 8 and following.

*This research was supported by the Polish Ministry of Education and Science grant no 1 P03B 075 29. 
The following problem is considered. In a spherically symmetric charged dust, just like in neutral dust, two kinds of singularity may be present: the Big Bang/Crunch $(\mathrm{BB} / \mathrm{BC})$, and shell crossings. Quite unlike in neutral dust, the $\mathrm{BB} / \mathrm{BC}$ singularity may be avoided. However, when the charge density $\rho_{e}$ and the mass-energy density $\epsilon$ obey $\left|\rho_{e}\right|<D_{e} \stackrel{\text { def }}{=} \sqrt{G} \epsilon / c^{2}$, the conditions that allow the model to avoid the BB/BC singularity necessarily lead to shell crossings. (The repulsive term that prevents the $\mathrm{BB} / \mathrm{BC}$ singularity in this case is a purely relativistic effect.) There exist initial conditions that allow us to avoid both singularities - when either $\left|\rho_{e}\right|>D_{e}$ everywhere, or $\left|\rho_{e}\right| \rightarrow D_{e}$ at the center of symmetry while $\left|\rho_{e}\right|<D_{e}$ elsewhere. The first case was considered by Ori [7], but remained unpublished. In the second case, both kinds of singularity may be avoided for a sufficiently long period that a body of charged dust goes through the tunnel between the singularities in the maximally extended Reissner - Nordström spacetime, to emerge into another asymptotically flat region. An explicit example of such a configuration is presented and discussed. It has $E<0$, i.e. it belongs to the recollapsing class that could possibly be pulsating for ever. However, shell crossings prevent it from existing for longer than one full cycle of collapse and expansion. As shown in the last section, the parameters of the object do not violate any conventional astrophysical limitations.

It is generally expected that shell crossings are just an artefact of the assumption that the spatial gradient of pressure is zero. In a more general solution, they would be replaced by acoustic waves of a high but finite density that become singularities in the limit of spatially homogeneous pressure (in particular, of zero pressure). However, no exact solutions of this kind are available, and the singularities in such configurations have so far been considered only via existence theorems applied to Einstein's equations with various sources, such as the Einstein - Maxwell - Vlasov equations treated in Ref. [8]. Typically, the existence theorems predict the properties of solutions over a rather limited time-interval. Lacking any more general exact solution, the charged dust models are the best device that is available for investigating long-term evolution of charged bodies.

\section{Spherically symmetric dust models with $R,_{r} \neq 0$}

With spherical symmetry, in the coordinates of (1.1), the electromagnetic tensor can have at most two nonzero components, $F_{t r}$ (the electric field) and $F_{\vartheta \varphi}$ (the magnetic field). The $F_{\vartheta \varphi} \neq 0$ is due to a distribution of magnetic monopoles. Since they are known (from experiment) not to exist, and since their inclusion does not lead to any significant generalisation 1 , we will assume the magnetic monopole charge to be zero everywhere.

The Einstein-Maxwell equations are in this case

$$
\begin{aligned}
R_{\alpha \beta}-\frac{1}{2} g_{\alpha \beta} R+\Lambda g_{\alpha \beta} & =\frac{8 \pi G}{c^{4}} \epsilon u_{\alpha} u_{\beta} \\
& +\frac{2 G}{c^{4}}\left(F_{\alpha}{ }^{\mu} F_{\mu \beta}+\frac{1}{4} g_{\alpha \beta} F_{\mu \nu} F^{\mu \nu}\right), \\
F^{\mu \nu}{ }_{i \nu} & =(4 \pi / c) \rho_{e} u^{\mu}, \\
F_{[\mu \nu, \rho]} & =0,
\end{aligned}
$$

\footnotetext{
${ }^{1}$ With magnetic monopoles taken into account, the electric charge $Q$ has to be replaced in all formulae by $\sqrt{Q_{e}{ }^{2}+Q_{m}^{2}}$, where $Q_{e}$ is the electric charge and $Q_{m}$ is the magnetic charge.
} 
where $\epsilon$ is the mass density of dust, $u^{\alpha}$ is the velocity field of dust, and $\rho_{e}$ is the density of the electric charge.

From (2.2) we find

$$
\begin{aligned}
F^{01} & =Q(r) \mathrm{e}^{-(A+C) / 2} / R^{2}, \\
Q, r & =(4 \pi / c) \rho_{e} \mathrm{e}^{A / 2} R^{2},
\end{aligned}
$$

where $Q(r)$ is an arbitrary function; it is the electric charge within the $r$-surface as (2.5) shows. With the assumed $F_{23}=0$, eq. (2.3) is fulfilled identically.

Now the coordinate components of the Einstein equations with the metric (1.1) and the electromagnetic tensor $(\underline{2.4})-(\underline{2.5})$ become

$$
\begin{aligned}
G_{00} & =\frac{8 \pi G}{c^{4}} \epsilon \mathrm{e}^{C}+\frac{G}{c^{4}} \frac{Q^{2}}{R^{4}} \mathrm{e}^{C}-\Lambda \mathrm{e}^{C}, \\
G_{01} & =0, \\
G_{11} & =-\frac{G}{c^{4}} \frac{Q^{2}}{R^{4}} \mathrm{e}^{A}+\Lambda \mathrm{e}^{A}, \\
G_{22} & \equiv G_{33} / \sin ^{2}(\vartheta)=\frac{G}{c^{4}} \frac{Q^{2}}{R^{2}}+\Lambda R^{2} .
\end{aligned}
$$

For $G_{\alpha \beta}$ we find

$$
\begin{aligned}
& G_{00}=\frac{\mathrm{e}^{C}}{R^{2}}+\frac{R_{, t}^{2}}{R^{2}}+\frac{A, t}{R} \\
& +\mathrm{e}^{C-A}\left(-\frac{R,_{r}^{2}}{R^{2}}-2 \frac{R,,_{r r}}{R}+\frac{A,_{r} R,,_{r}}{R}\right), \\
& G_{01}=-2 \frac{R, t r}{R}+\frac{A,{ }_{t} R,,_{r}}{R}+\frac{R,{ }_{t} C,_{r}}{R}, \\
& G_{11}=-\frac{\mathrm{e}^{A}}{R^{2}}+\frac{R_{, r}^{2}}{R^{2}}+\frac{C_{,_{r}} R,_{r}}{R} \\
& +\mathrm{e}^{A-C}\left(-\frac{R, t}{R^{2}}-2 \frac{R, t t}{R}+\frac{C,{ }_{t} R, t}{R}\right), \\
& G_{22}=\frac{1}{4} \mathrm{e}^{-C}\left(-4 R R,{ }_{t t}+2 R C,_{t} R,_{t}-2 R A,_{t} R,_{t}\right. \\
& \left.-R^{2} A,_{t}^{2}-2 R^{2} A, t t+R^{2} C,_{t} A, t\right) \\
& +\frac{1}{4} \mathrm{e}^{-A}\left(4 R R,_{r r}+2 R C,_{r} R,_{r}-2 R A,_{r} R,_{r}\right. \\
& \left.+R^{2} C_{, r}^{2}+2 R^{2} C_{, r r}-R^{2} C_{,_{r}} A,_{r}\right) .
\end{aligned}
$$

Equations (2.1) - (2.3) imply the following:

$$
\begin{aligned}
\left(\epsilon u^{\beta}\right) ;_{\beta} & =0 \\
\epsilon u^{\alpha}{ }_{\beta} u^{\beta} & =-\frac{1}{c} \rho_{e} F^{\alpha}{ }_{\beta} u^{\beta} .
\end{aligned}
$$

Equations (2.14) (the conservation of mass) and (2.15) (the Lorentz force acting on charges in motion and pushing them off geodesic trajectories) are quite general and independent 
of any symmetry properties of spacetime. Applying (2.15) to our metric (1.1) we get

$$
\epsilon,_{, r}=\frac{1}{2 \pi} \frac{Q Q,,_{r}}{R^{4}} .
$$

Applying (2.14) to our metric (1.1) we then get

$$
\frac{\kappa}{2} \epsilon R^{2} \mathrm{e}^{A / 2}=\frac{G}{c^{4}} N_{, r},
$$

where $N_{, r}$ is an arbitrary function of integration. We see that $N$ so defined is the energy equivalent to the sum of rest masses within the $r$-surface. From (2.5) and (2.17) we now see that the ratio $Q, r / N_{, r}=\rho_{e} /(c \epsilon)$ is time-independent.

Using now (2.16) and (2.11) we obtain from the equation $G_{01}=0$

$$
2 \mathrm{e}^{-A / 2} R_{t_{r}}-\mathrm{e}^{-A / 2} A,_{t} R,_{r}=\frac{2}{c} \frac{R, t}{R^{2}}\left(\frac{\rho_{e} Q}{\epsilon}\right) .
$$

(It is here that the case $R_{r}=0$ has to be set aside for separate investigation. With $R, r=0$, the equation $G_{01}=0$ reduces to $R,{ }_{t} C,_{r}=0$ and cannot be used to determine $R, r_{r}$ as we do below.) Since the expression in parentheses is independent of $t$, this can be integrated with the result

$$
\mathrm{e}^{-A / 2} R, r=\Gamma(r)-\frac{\rho_{e} Q}{c \in R},
$$

where $\Gamma(r)$ is an arbitrary function of integration. Using (2.5) and (2.17) we now find

$$
\frac{\rho_{e} Q}{c \epsilon} \equiv \frac{Q Q, r}{N, r}=Q Q,_{N} .
$$

With this, (2.19) becomes

$$
\mathrm{e}^{-A / 2} R, r=\Gamma(r)-\frac{Q Q,_{N}}{R},
$$

and now (2.16) becomes

$$
C_{, r}=2 \frac{\mathrm{e}^{A / 2}}{R^{2}} Q Q,_{N} .
$$

Using (2.21) and (2.22) to eliminate $R_{, r}$ and $C,,_{r}$ from (2.12) and integrating the $G_{11}$ equation we get

$$
\mathrm{e}^{-C} R,{ }_{t}{ }^{2}=\Gamma^{2}-1+\frac{2 M(r)}{R}+\frac{Q^{2}\left(Q,,_{N}^{2}-G / c^{4}\right)}{R^{2}}-\frac{1}{3} \Lambda R^{2},
$$

where $M(r)$ is an arbitrary function. Comparing this with the Newtonian equation of motion we see that $\left(\Gamma^{2}-1\right) / 2$ plays here the role of the energy function $E(r)$.

The function $M(r)$ is the effective mass that, together with $Q$, drives the evolution. However, as we will see, $M(r)$ is a combination of mass and charge that need not be positive. In order to see this, we compare (2.23) with the Newtonian limit, assuming $\Lambda=0$. Let $[x]$ denote the physical dimension (unit) of $x$. The dimensions of the quantities appearing in (2.23) are: $[R]=[$ length $],\left[\mathrm{e}^{C / 2} \mathrm{~d} t\right] \equiv[\mathrm{d} s]=[c] \times[$ time $],[M]=[G] \times[$ mass $] /\left[c^{2}\right]$, $[N]=[$ mass $] \times\left[c^{2}\right],[Q]=[$ charge $] \equiv[\sqrt{\text { mass }}] \times\left[\right.$ length $\left.^{3 / 2}\right] /[$ time $]$. The function $\left(\Gamma^{2}-1\right)$ 
is dimensionless, but, for consistency, must be assumed to have the form $2 \mathcal{E} / c^{2}$, where $[\mathcal{E}]=\left[\right.$ velocity $\left.^{2}\right]$. In order to find the Newtonian limit of (2.23), we multiply it by $c^{2}$ and let $c \rightarrow \infty$. Denoting the Newtonian time by $\tau$ we obtain

$$
R,_{\tau}^{2}=2 \mathcal{E}+2 G m(r) / R .
$$

But the Newtonian equation of motion of spherically symmetric charged dust is

$$
r_{, \tau}{ }^{2}=2 \mathcal{E}(r)+2\left[G \mathcal{M}(r)-\frac{\rho_{e}(r)}{\rho_{\mu}(r)} Q(r)\right] / r,
$$

where $\mathcal{M}(r)$ is the total mass within the sphere of radius $r, Q(r)$ is the total charge within the same sphere, $\rho_{e}$ is the charge density and $\rho_{\mu}$ is the mass density. Thus, the $M(r)$ in (2.23) corresponds to the Newtonian $\left[G \mathcal{M}(r)-\left(\rho_{e}(r) / \rho_{\mu}(r)\right) Q(r)\right]$, and, as announced, does not have to be positive.

To verify the $G_{22}$ equation, we have to find $C_{, t}$ from the $G_{11}$ equation and $A_{, t}$ from the $G_{01}=0$ equation. Substituting these, then finding $A, r$ from (2.21), using the $r$-derivative of (2.23) to eliminate $R_{t} R_{, t r}$, and again using (2.21) to eliminate $\mathrm{e}^{-A}$, we obtain

$$
Q Q,{ }_{N}\left[-\frac{G}{c^{4}} \Gamma N_{, r}+\left(M+Q Q,_{N} \Gamma\right){ }_{, r}\right]=0 .
$$

One solution of this is $Q,_{N}=0$, i.e. a constant total charge. We will mention this simpler case later. When $Q,_{N} \neq 0$,

$$
\frac{G}{c^{4}} \Gamma N,_{r}=\left(M+Q Q,_{N} \Gamma\right){ }_{{ }_{r}} .
$$

The quantity

$$
\mathcal{M} \stackrel{\text { def }}{=} M+Q Q, N \Gamma,
$$

an exact analogue of the Newtonian $\mathcal{M}(r)$ of (2.25), will appear again in Sec. 3. There we will find that $\mathcal{M}$ is the active gravitational mass. Thus, via (2.27), $\Gamma$ determines by how much $\mathcal{M}$ increases when a unit of rest mass is added to the source, i.e. $\Gamma$ is a measure of the gravitational mass defect/excess. Solutions with $\Gamma=0$ are known, this is the Datt - Ruban class [1] - [4]. Negative $\Gamma$ can also occur (see subsection 18.20.2 of Ref. [6]). However, the case $\Gamma>0$ corresponds to the most ordinary configuration.

The final equation to take into account is (2.6), and it reproduces (2.17). Using (2.21) to eliminate $\mathrm{e}^{A / 2}$ from (2.17), we get $\epsilon$ in an equivalent form:

$$
\kappa \epsilon=\frac{2 G N_{, r}}{c^{4} R^{2} R,_{r}}\left(\Gamma-\frac{Q Q,_{N}}{R}\right) .
$$

Finally, the Einstein-Maxwell equations for charged dust reduced to a set that defines the functions $A(t, r), C(t, r)$ and $R(t, r)$ implicitly. The solution is constructed as follows:

1. Choose the functions $N(r), Q(r)$ and $\Gamma(r)$, and then solve (2.27) to find $M(r)$. In fact, since the coordinate $r$ is determined up to the transformations $r^{\prime}=f(r)$, where $f$ is an arbitrary function, we can choose $N, Q$ or $\Gamma$ as the radial coordinate. In the example in Sec. 11, our radial coordinate will be $N(r)$. 
2. Given these, express $\mathrm{e}^{A / 2}$ through $R$ via (2.21).

3. The set of equations (2.22) - (2.23) then defines $\mathrm{e}^{C}$ and $R$. In solving (2.22) numerically for $C(t, r)$, an initial condition has to be assumed. Note that so far $C$ is not defined uniquely - the coordinate $t$ can be transformed by $t=g\left(t^{\prime}\right)$, and then $C$ transforms by $C=C^{\prime}-2 \ln \left(g_{, t^{\prime}}\right)$. Using this, we can require that at the center of symmetry $C\left(t, r_{c}\right)=0$, i.e. for the particle that remains all the time at the center the proper time $s=t$ - the time coordinate in spacetime.

If $Q,{ }_{N}=0$, then $Q=$ const, i.e. $\rho_{e}=0$ from (2.5). This case is the neutral dust moving in the exterior electric field of a charge concentrated at $R=0$.

With vanishing charge, eq. (2.15) reduces to the equation of a geodesic, while eqs. (2.21), (2.22) and (2.23) reduce to those defining the the Lemaître-Tolman model [5, 6].

\section{Matching the charged dust metric to the Reissner- Nordström metric}

We first transform the $\mathrm{R}-\mathrm{N}$ metric with $\Lambda$ to the appropriate coordinates. For the standard curvature coordinates we write $\left(\tau, R_{R N}\right)$, and we introduce the symbol

$$
h \stackrel{\text { def }}{=} 1-\frac{2 m}{R_{R N}}+\frac{e^{2}}{R_{R N}{ }^{2}}+\frac{1}{3} \Lambda R_{R N}{ }^{2} .
$$

We demand that the new coordinates $(t, r)$ are still orthogonal, so that $g_{t r}=0$, i.e.

$$
h{,{ }_{t}} \tau_{, r}-\frac{1}{h} R_{R N, t} R_{R N, r}=0 .
$$

The function $C_{R N}(t, r)$ is

$$
\mathrm{e}^{C_{R N}}=h \tau, t^{2}-\frac{1}{h} R_{R N, t^{2}}{ }^{2}
$$

We now solve (3.2) - (3.3) for $\tau_{r}$ and calculate

$$
\left(g_{11}\right)_{R N} \equiv h \tau, r^{2}-\frac{1}{h} R_{R N, r}{ }^{2}=-\frac{\mathrm{e}^{C} R_{R N, r}{ }^{2}}{h \mathrm{e}^{C}+R_{R N, t}{ }^{2}} .
$$

The component $\left(g_{00}\right)_{R N}$ is not fully determined at this point, we will determine it later. Since $\tau$ is defined by the partial differential equation (3.2), it still involves an arbitrary function of one variable.

We wish to match the charged dust metric of Sec. 1 to the $\mathrm{R}-\mathrm{N}$ solution given above across a hypersurface $r=r_{b}$. This requires that the induced 3-metric and the second fundamental form of this hypersurface are the same for both spacetime metrics. Continuity of the 3-metric requires that

$$
\mathrm{e}^{C\left(t, r_{b}\right)}=\mathrm{e}^{C_{R N}\left(t, r_{b}\right)}, \quad R\left(t, r_{b}\right)=R_{R N}\left(t, r_{b}\right) .
$$

The transformations that keep the metric diagonal are still allowed. Transforming $t$ by $t^{\prime}=\int \mathrm{e}^{\alpha(t)} \mathrm{d} t$, where $\alpha=C_{R N}\left(t, r_{b}\right)-C\left(t, r_{b}\right)$ we fulfil the first of (3.5), while $g_{11}^{\prime}$ and $g_{01}^{\prime}$ are not changed. 
On the surface $r=r_{b}, R_{R N}\left(t, r_{b}\right)$ must be the same function of $t$ as $R\left(t, r_{b}\right)$. Consequently, $R, t\left(t, r_{b}\right)$ must be the same in both metrics, and so $R_{R N}\left(t, r_{b}\right)$ must obey

$$
\begin{gathered}
\mathrm{e}^{-C_{R N}\left(t, r_{b}\right)} R_{R N, t}{ }^{2}\left(t, r_{b}\right)=\Gamma^{2}\left(r_{b}\right) \\
-1+\frac{2 M\left(r_{b}\right)}{R_{R N}\left(t, r_{b}\right)}-\frac{G Q^{2}\left(r_{b}\right)}{c^{4} R_{R N}{ }^{2}\left(t, r_{b}\right)} \\
-\frac{1}{3} \Lambda R_{R N}{ }^{2}\left(t, r_{b}\right) .
\end{gathered}
$$

The unit vector normal to the hypersurface $r=r_{b}$ has components

$$
X^{\mu}=\left(0, \mathrm{e}^{-A / 2}, 0,0\right) \equiv\left(0,\left(\Gamma-\frac{Q Q,_{N}}{R}\right) / R,_{r}, 0,0\right)
$$

for the interior metric, and, from (3.4),

$$
X_{R N}^{\mu}=\left(0, \frac{\sqrt{h+\mathrm{e}^{-C_{R N} R_{R N, t}{ }^{2}}}}{R_{R N, r}}, 0,0\right)
$$

for the $\mathrm{R}-\mathrm{N}$ solution. From the continuity of the second fundamental form we have $R,\left._{r} X^{r}\right|_{r=r_{b}}=\left.R_{R N, r} X_{R N}^{r}\right|_{r=r_{b}}$ and $\left.\left(\mathrm{e}^{C}\right){ }_{r} X^{r}\right|_{r=r_{b}}=\left(\mathrm{e}^{C_{R N}}\right),\left._{r} X_{R N}^{r}\right|_{r=r_{b}}$. The first condition says

$$
\left(h+\mathrm{e}^{-C_{R N}} R_{R N, t}^{2}\right)_{r=r_{b}}=\left(\Gamma-\frac{Q Q, N}{R}\right)_{r=r_{b}}^{2},
$$

which ensures the continuity of $g_{11}=-\mathrm{e}^{A}$ across $r=r_{b}$, even though we have not required this. Substituting for $R_{R N, t}{ }^{2}$ from (3.6) and for $h$ from (3.1), then comparing the coefficients we obtain

$$
e=\frac{\sqrt{G}}{c^{2}} Q\left(r_{b}\right), \quad m=\left(M+Q Q,_{N} \Gamma\right)_{r=r_{b}} .
$$

The continuity of the second fundamental form imposes one last condition, on $C,_{r}$. Using (2.22), (2.21) and (3.4), the condition is

$$
\left.C_{R N, r} \frac{\Gamma-Q Q,_{N} / R}{R_{R N, r}}\right|_{r=r_{b}}=2 \frac{Q Q,_{N}}{R^{2}} .
$$

We have no expression yet for $C_{R N, r}$, and we will find it from the field equations now. We know that $\mathrm{e}^{A}$ is continuous at $r=r_{b}$, so we can use (2.21) for $A_{R N}\left(t, r_{b}\right)$. Substitute this in (2.11), and take the equation $G_{01}=0$ at $r=r_{b}$. The result is

$$
C_{R N, r}\left(t, r_{b}\right)=\left.\frac{2 R_{R N, r} Q Q,_{N}}{R^{2}\left(\Gamma-Q Q,_{N} / R\right)}\right|_{r=r_{b}},
$$

and it shows that (3.11) is fulfilled.

Thus, (3.10) are the only limitations imposed on the charged dust metric by the matching conditions. This matching was first discussed by Vickers [9]. The second of (3.10) reveals the connection between the active gravitational mass (in this case $m$ ) and the effective mass (compare (2.28) $)$. 


\section{Prevention of the Big Crunch singularity by electric charge}

In the present section we will deal only with the Big Bang/Big Crunch singularities, assuming $\Lambda=0$. The shell crossings will be discussed separately in Sec. 17. In the following we shall denote $E(r)=\left(\Gamma^{2}-1\right) / 2$.

The presence or absence of a singularity is detected by investigating the roots of the right-hand side of (2.23), which, for this purpose, is more conveniently written as

$$
\mathrm{e}^{-C} R^{2} R,{ }_{t}^{2}=2 E(r) R^{2}+2 M(r) R+Q^{2}\left(Q,{ }_{N}{ }^{2}-G / c^{4}\right) \stackrel{\text { def }}{=} W(R) .
$$

At each root of $W(R)$, the sign of $R, t$ changes, and evolution is possible only in those regions where $W(R) \geq 0$. The following cases occur

(a) When $E<0, W(R)$ has roots only if

$$
M^{2} \geq 2 E Q^{2}\left(Q,,_{N}^{2}-G / c^{4}\right) .
$$

With no roots, $W(R)$ would be negative at all $R$, so (4.2) is the condition for the existence of a solution of (4.1). The roots are

$$
R_{ \pm}=-\frac{M}{2 E} \pm \frac{1}{2 E} \sqrt{M^{2}-2 E Q^{2}\left(Q,,_{N}^{2}-G / c^{4}\right)}
$$

and $W(R)>0$ between them. Nonsingular solutions will exist when both $R_{ \pm}>0$ (with $R_{ \pm}<0$, no solution exists at all, and with $R_{-} R_{+}<0, R=0$ is in the allowed range.) This is equivalent to

$$
Q,,_{N}^{2}<G / c^{4} \quad \text { and } \quad M>0 .
$$

We will interpret this condition later on in this section.

If there is equality in (4.2), then $W(R)<0$ for all $R \neq R_{-}=R_{+}$, and $W\left(R_{ \pm}\right)=0$. Then $R=R_{ \pm}$and the model is static. If, in addition, $Q,_{N}\left(r_{b}\right)=0$ (meaning $\rho_{e}\left(r_{b}\right)=0$ ) and $\Gamma\left(r_{b}\right)=0$, then $E=-1 / 2$, and in this case the exterior $\mathrm{R}-\mathrm{N}$ metric is the extreme one, with $e^{2}=m^{2}$.

With (4.4) fulfilled, $R$ oscillates between a nonzero minimum and a maximum.

(b) When $E=0$, singularity is avoided if and only if $M>0$ and $Q,{ }_{N}{ }^{2}<G / c^{4}$. Collapse is then halted and reversed once and for all.

(c) When $E>0, W(R)>0$ either everywhere (if there are no roots) or beyond the

roots. There will be no roots when $M^{2}<2 E Q^{2}\left(Q,{ }_{N}{ }^{2}-G / c^{4}\right)$, in which case $W(R)>0$ for all $R$ including $R=0$, and the model can run into the singularity. Thus (4.2) is here one of the necessary conditions for the existence of nonsingular solutions. With (4.2) fulfilled, $W(R)$ has two roots, and at least one of them has to be positive if singularity is to be avoided. With $M>0$, we have $R_{-}<0$ always and $R_{+}>0$ if and only if $Q,{ }_{N}{ }^{2}<G / c^{4}$. With $M<0, R_{+}>0$ and $R_{-}<R_{+}$always, so nonsingular solutions exist with no further conditions, provided $R>R_{+}$initially. Collapse is then halted and reversed as in case (b). The bounce with $M<0$ is nonrelativistic, since it occurs also in Newton's theory, under the same conditions.

Now we will interpret the condition (4.4). The inequality $Q,{ }_{N}^{2}<G / c^{4}$ translates into $\left|\rho_{e}\right|<\sqrt{G} \epsilon / c$, which means that the absolute value of the charge density is sufficiently small 
(but nonzero) compared to the mass density. This kind of bounce is purely relativistic, and it does not occur in the Newtonian limit: with $M>0, R=0$ is always in the allowed range of Newtonian solutions. The interpretation of the relativistic bounce in the Newtonian terms is this: as seen from (2.23), the charges provide a correction to the effective mass $M$, so that it becomes $\bar{M}=M+(1 / 2) Q^{2}\left(Q,{ }_{N}{ }^{2}-G / c^{4}\right) / R$. This correction is negative at small charge density (when $Q,{ }_{N}^{2}<G / c^{4}$ ), so it weakens gravitation, thus helping the dust to bounce. However, at large charge density $\left(Q,,_{N}^{2}>G / c^{4}\right)$, charges enhance the effective mass and thus oppose bounce. (A similar phenomenon is encountered in the motion of particles in the Reissner-Nordström spacetime, where an electric charge in the source of the gravitational field creates effective antigravitation, provided the charge is small enough compared to mass.) Nevertheless, in this last case, the Newtonian electrostatic repulsion can prevail, provided $M<0$ at the same time.

With $Q,_{N}=0$ the $\mathrm{BB} / \mathrm{BC}$ singularity is avoided in every case when a solution exists. Thus, for neutral dust moving in an exterior electric field, the BB/BC singularity never occurs. This was first found by Shikin [10]. This is a purely relativistic effect.

The above implies that with (4.4) fulfilled a solution of (2.23), for which $R \neq 0$ initially, does not go down to 0 . However, if the charged dust occupies a volume around the center of symmetry $R=0$, then, at any time, there are dust particles with all values of $R$, including $R=0$. (We will find in the next section the conditions to be obeyed in order that the center is nonsingular.) Thus, the inner turning points given by (4.3) will exist arbitrarily close to the center. We will use this remark in Sec. 7.

If $E M<0$ and $Q,{ }_{N}^{2}=G / c^{4}$, then (4.1) has the time-independent solution $R=-M / E$. In this case, the electrostatic repulsion just balances the gravitational attraction and the whole configuration is static - but unstable. When $M>0>E$, the perturbation can only be toward smaller $R$, and it will send the dust into collapse that will terminate at $R=0$. When $M<0<E$, the perturbation can only be toward larger $R$, and it will send the dust into infinite expansion. Another time-independent solution is $E=0=M, Q,{ }_{N}{ }^{2}=G / c^{4}$; in this case $G \Gamma / c^{4}=D=$ const and $N=D \mathcal{M}$.

The surface of the charged sphere obeys the equation of radial motion of a charged particle in the Reissner-Nordström spacetime. For such a particle, if the ratio of its charge $q$ to its mass $\mu$ obeys $(q / \mu)^{2}<1$, then the reversal of fall to escape can occur only inside the inner $\mathrm{R}-\mathrm{N}$ horizon, at $R<r_{-}=m-\sqrt{m^{2}-e^{2}}$. Thus, the surface of a collapsing sphere must continue to collapse until it crosses the inner horizon, and can bounce at $R<r_{-}$. Then, however, it cannot re-expand back into the same spacetime region from which it collapsed, as this would require motion backward in time, as seen from the compactified spacetime diagram of the maximally extended $\mathrm{R}-\mathrm{N}$ solution [6]. The surface would thus continue through the tunnel between the singularities and re-expand into another copy of the asymptotically flat region. This possibility is interesting, since it shows that the maximally extended $\mathrm{R}-\mathrm{N}$ spacetime, usually interpreted as an abstract geometric curiosity, may in this case become an astrophysical reality.

The bounce at small charge density $\left(Q,{ }_{N}{ }^{2}<G / c^{4}\right)$ is more interesting physically, since the real Universe has no detectable net charge, so only small localized charges could exist in it. We saw that an arbitrarily small uncompensated charge can prevent the $B B / B C$ singularity. Unfortunately, Ori [11, 12] proved that if $Q,,_{N}^{2}<G / c^{4}$ holds throughout the volume, then a shell crossing is unavoidable, and it will block the passage through the tunnel. We will derive this result in Sec. 7. Thus, a nonsingular bounce through the RN 
tunnel is possible only if $Q, N^{2}>G / c^{4}$ everywhere or if $Q, N^{2} \rightarrow G / c^{4}$ at the center, while $Q,,_{N}^{2}<G / c^{4}$ elsewhere.

\section{Charged dust in curvature and mass-curvature co- ordinates}

It is instructive to transform the metric given by (1.1) with (2.21) - (2.23) to such coordinates in which the function $R(t, r)$ is the radial coordinate. We note that $R,{ }_{r} \mathrm{~d} r=$ $\mathrm{d} R-R, t \mathrm{~d} t$, and we take $t$ to be a function of the new coordinates: $t=f(\tau, R)$. Thus

$$
\begin{aligned}
R,_{r} \mathrm{~d} r & =\mathrm{d} R-R,,_{t}\left(f,_{\tau} \mathrm{d} \tau+f,_{R} \mathrm{~d} R\right) \\
\mathrm{d} t & =f,_{\tau} \mathrm{d} \tau+f,_{R} \mathrm{~d} R
\end{aligned}
$$

and the new metric components, using (2.23), are found to be

$$
\begin{aligned}
g_{\tau \tau} & =\mathrm{e}^{C} f,_{\tau}{ }^{2} \Delta /\left(\Gamma-Q Q,_{N} / R\right)^{2}, \\
g_{\tau R} & =\frac{\mathrm{e}^{C} f,_{\tau} f,_{R} \Delta+f,_{\tau} R,_{t}}{\left(\Gamma-Q Q,_{N} / R\right)^{2}}, \\
g_{R R} & =\frac{\mathrm{e}^{C} f,_{R}{ }^{2} \Delta-1+2 f,_{R} R, t}{\left(\Gamma-Q Q,_{N} / R\right)^{2}}, \\
\Delta & \stackrel{\text { def }}{=} 1-\frac{2 \mathcal{M}}{R}+\frac{G Q^{2}}{c^{4} R^{2}}+\frac{1}{3} \Lambda R^{2},
\end{aligned}
$$

where $\mathcal{M}$ is defined in (2.28). Note, from (5.1), that the transformation is different for collapsing dust $(R, t<0)$ and for expanding dust $(R, t>0)$. The transformation from $(\tau, R)$ to $(t, r)$, inverse to (5.1), is analogous to introducing, in the Reissner-Nordstöm region $r>r_{+}=m+\sqrt{m^{2}-e^{2}}$, coordinates comoving with the congruence of charged particles that are radially collapsing or expanding, respectively. The extension is to the future or to the past, respectively.

This can now be specialised in two ways. One possibility is to choose the proper curvature coordinates, in which $g_{\tau R}=0$. This representation of the Vickers metric has not found any application in the literature so far, but it is instructive - see Ref. [6]. The other possible specialisation of (5.2) - (5.4) is to choose the $\mathcal{M}$ defined in (2.28) as the new $\tau$ coordinate. These mass-curvature coordinates were first introduced by Ori [11]. The surfaces $\mathcal{M}=$ const are timelike, so none of the coordinates is time and the metric in these coordinates cannot be diagonal. Since $\mathcal{M}, Q, \Gamma$ and $N$ depend only on $r$ in the original coordinates, we have $Q=Q(\mathcal{M}), \Gamma=\Gamma(\mathcal{M})$ and $N=N(\mathcal{M})$. The Jacobi matrices of the transformations $(t, r) \leftrightarrow(\mathcal{M}, R)$ are

$$
\frac{\partial(t, r)}{\partial(\mathcal{M}, R)}=\left[\begin{array}{ll}
f, \mathcal{M}, & f,_{R} \\
r, \mathcal{M}, & r, R
\end{array}\right], \quad \frac{\partial(\mathcal{M}, R)}{\partial(t, r)}=\left[\begin{array}{ll}
0 & \mathcal{M},_{r} \\
R, t & R,_{r}
\end{array}\right]
$$

These matrices must be inverse to each other, hence

$$
f,_{\mathcal{M}}=-\frac{R,_{r}}{R, \mathcal{M}_{, r}}, \quad f,_{R}=1 / R,_{t}, \quad r, \mathcal{M}=1 / \mathcal{M}_{r}, \quad r,_{R}=0
$$


In the coordinates $\left(x^{0}, x^{1}\right) \stackrel{\text { def }}{=}(\mathcal{M}, R)$ the velocity field still has only one contravariant component:

$$
u^{R}= \pm \sqrt{\Gamma^{2}-1+\frac{2 M}{R}+\frac{Q^{2}\left(Q, N^{2}-G / c^{4}\right)}{R^{2}}-\frac{1}{3} \Lambda R^{2}}
$$

(+ for expansion, - for collapse). We define the auxiliary quantities

$$
\begin{gathered}
u \stackrel{\text { def }}{=} \Gamma-Q Q,_{N} / R, \\
\left(\mathrm{e}^{C / 2} / u\right) f, \mathcal{M} \stackrel{\text { def }}{=} F(\mathcal{M}, R),
\end{gathered}
$$

and, using (5.5), (5.8) and (2.23), we get in (5.2) - (5.4):

$$
g_{\mathcal{M M}}=F^{2} \Delta, \quad g_{\mathcal{M R}}=F u / u^{R}, \quad g_{R R}=1 /\left(u^{R}\right)^{2},
$$

while eqs. (5.1) simplify to $\mathrm{d} r=\mathrm{d} \mathcal{M} / \mathcal{M},_{r}$ and $\mathrm{d} t=\left(-R, r \mathrm{~d} \mathcal{M} / \mathcal{M},_{r}+\mathrm{d} R\right) / R,{ }_{t}$. The function $F(\mathcal{M}, R)$ is to be found from the field equations.

Using (5.7), (2.4) and (5.10) we find that the only nonvanishing components of the electromagnetic tensor in the $(\mathcal{M}, R)$ coordinates are

$$
F^{\mathcal{M} R}=-F^{R \mathcal{M}}=\frac{Q}{F R^{2}}, \quad F_{\mathcal{M} R}=-F_{R \mathcal{M}}=-\frac{F Q}{R^{2}} .
$$

Further, using (2.4), (2.5), (2.17), (2.21), (2.27), (2.23) and (5.8) we find for the charge density and energy-density

$$
\frac{4 \pi \rho_{e}}{c}=-\frac{Q, \mathcal{M}}{R^{2} F u^{R}}, \quad \kappa \epsilon=-\frac{2}{\Gamma R^{2} F u^{R}} .
$$

Recall that the $(\mathcal{M}, R)$-coordinates cover only such a region where $R,{ }_{t}$ has a constant sign. As seen from (5.7) and (5.10), $F$ changes sign where $R,{ }_{t}$ does, and so does $u^{R}=$ $\mathrm{e}^{-C / 2} R, t$. Thus, $F u^{R}$ preserves its sign when collapse turns to expansion and vice versa. This observation will be useful when calculating $F$ later.

Now $F(\mathcal{M}, R)$ is the only unknown function. We obtain further

$$
\begin{aligned}
u_{\mathcal{M}} & =u F, \quad u_{R}=1 / u^{R}, \\
g^{\mathcal{M M}} & =-\frac{1}{\left(F u^{R}\right)^{2}}, \quad g^{\mathcal{M} R}=\frac{u}{F u^{R}}, \quad g^{R R}=-\Delta .
\end{aligned}
$$

In the $(\mathcal{M}, R)$ coordinates eq. (2.27) reads

$$
\left(G \Gamma / c^{4}\right) N,_{\mathcal{M}}=1 .
$$

The function $F$ can be found from (2.15). Using (2.20), it is found to be:

$$
F, R=-\frac{u^{R}, \mathcal{M}}{u\left(u^{R}\right)^{2}} .
$$

Using (5.9) and (5.16) we transform (5.17) to

$$
F,_{R}=-\frac{1}{\left(u^{R}(\mathcal{M}, R)\right)^{3}}\left\{\Gamma,_{\mathcal{M}}+\frac{1}{R \Gamma}\left[1-\frac{c^{4}}{G}\left(Q,,_{N}^{2}+Q Q,,_{N}\right)\right]\right\} .
$$


This coincides, except for notation, with Ori's (1990) result. As Ori [11] stressed, eqs. (5.11), (5.8) and (5.18) determine the metric explicitly, in contrast to the representation by Vickers used in Sec. 2, where the Einstein-Maxwell equations were reduced to a set of two differential equations. However, we lost the information about the time-dependence of $R$. Points of the spacetime are now identified by the values of $\mathcal{M}$ and $R$ - by specifying the pair $(\mathcal{M}, R)$ we say: this is the point in which the shell containing the mass $\mathcal{M}$ has the radius $R$. However, we have no means of saying, without recourse to the comoving coordinates, how much coordinate time it has taken the shell to expand from the minimal size $R=R_{\text {min }}$ to the current $R$. In consequence of this, the $(\mathcal{M}, R)$ coordinates do not allow us to see whether the minimal size (which is a singularity or a nonsingular bounce) was achieved by all shells simultaneously with respect to the time coordinate $t$ or not. This information is crucial for considering shell crossings, as we will see in Sec. 7.

With $\Lambda=0$, the integral of (5.18) is elementary, but requires separate treatment of various subcases. The full list of results is given in Ori's paper.

Now the Einstein-Maxwell equations are all fulfilled.

Note from (5.11) and (5.13) that the metric and the mass density are insensitive to the sign of $u^{R}$. However, as explained in the remark after (5.5),$u^{R}>0$ and $u^{R}<0$ correspond to different maps with different domains. Thus, integrating (5.18) from $R_{1}$ to $R>R_{1}$ with $u^{R}>0$, we integrate forward in time, while calculating the same integral with $u^{R}<0$ we integrate backward in time.

\section{Regularity conditions at the center}

Just as in the $\mathrm{L}-\mathrm{T}$ model, the set $R=0$ in charged dust consists of the Big Bang/Crunch singularity (which we showed to be avoidable) and of the center of symmetry, which may or may not be singular. We will now derive the conditions for the absence of the central singularity. We assume no magnetic charges.

Let $r=r_{c}$ correspond to the center of symmetry. From (1.1), (2.21) and (2.29) we see

that $N(r)=\int_{\mathcal{V}} \epsilon \sqrt{-g} \mathrm{~d}_{3} x=4 \pi \int_{0}^{r} \epsilon\left(r^{\prime}\right) R^{2}\left(t, r^{\prime}\right) \mathrm{d} r^{\prime}$, where $\mathcal{V}$ is a sphere centered at $r=r_{c}$ in a $t=$ const space. Thus, if $\epsilon$ has no singularity of the type of the Dirac delta at the center, $N(r)$ must obey $N\left(r_{c}\right)=0$. Similarly, eqs. (1.1) and (2.5) show that if there is no delta-type singularity of $\rho_{e}$ at the center, then the electric charge must obey $Q\left(r_{c}\right)=0$. With both $\epsilon$ and $\rho_{e}$ being nonsingular at $r_{c}$, and $\epsilon\left(t, r_{c}\right)>0$, the ratio $\rho_{e}\left(r_{c}\right) / \epsilon\left(r_{c}\right)$ is nonsingular, and (2.5) with (2.17) show that $\lim _{r \rightarrow r_{c}} Q / N=\lim _{r \rightarrow r_{c}} Q,{ }_{N}=\rho_{e}\left(r_{c}\right) /\left[c \epsilon\left(r_{c}\right)\right]$ is finite (possibly zero). Then, (5.16) implies that $\Gamma\left(r_{c}\right) \neq 0$ and $\lim _{r \rightarrow r_{c}} N / \mathcal{M}=\lim _{r \rightarrow r_{c}} N, \mathcal{M}=G \Gamma / c^{4}$, and this, together with $N\left(r_{c}\right)=0$, implies $\mathcal{M}\left(r_{c}\right)=0$. Then, from (2.28), also $M\left(r_{c}\right)=0$.

Since $R\left(t, r_{c}\right)=0$ and $N\left(r_{c}\right)=0$, we find from (2.29):

$$
\lim _{r \rightarrow r_{c}} \frac{R^{3}}{N}=\lim _{r \rightarrow r_{c}} \frac{3 R^{2} R,,_{r}}{N,_{r}}=\lim _{r \rightarrow r_{c}} \frac{3}{4 \pi \epsilon}\left(\Gamma-\frac{Q Q,_{N}}{R}\right) .
$$

This limit will be finite if $\lim _{r \rightarrow r_{c}}\left(Q Q,_{N} / R\right)<\infty$. We already know that $Q,_{N}\left(r_{c}\right) \stackrel{\text { def }}{=} \widetilde{q}_{0}=$ const and thus $\lim _{N \rightarrow 0} Q / N=\widetilde{q}_{0}$. Thus, $\lim _{r \rightarrow r_{c}} Q / \mathcal{M}=q_{0}$ and $\lim _{r \rightarrow r_{c}}\left(Q Q,_{N} / R\right)<\infty$ if $\lim _{r \rightarrow r_{c}} R / \mathcal{M}^{\gamma}=$ const, where $\gamma<1$. Then, (6.1) imposes the further condition that, in the neighbourhood of $r_{c}$

$$
\lim _{r \rightarrow r_{c}} R / \mathcal{M}^{1 / 3}=\beta(t)
$$


We assume $\beta(t) \neq 0$, since $\beta=0$ implies, via (6.1), one of two unphysical situations: (I) $\lim _{r \rightarrow r_{c}} \epsilon=\infty$, i.e. a permanent central singularity, or (II) $\lim _{r \rightarrow r_{c}}\left(\Gamma-Q Q,,_{N} / R\right)=0$, which leads to $\epsilon\left(r_{c}\right)$ being independent of time - a pathological situation in an expanding or contracting configuration.

Since $R\left(t, r_{c}\right)=0$ at all times, we have $R_{t}\left(t, r_{c}\right)=0$, and, from the above, $\lim _{r \rightarrow r_{c}}$ $R,{ }_{t} / \mathcal{M}^{1 / 3}=\beta,_{t}$. All other terms in (2.23) except $\left(\Gamma^{2}-1\right)$ vanish at $r=r_{c}$, and so we must have

$$
\lim _{r \rightarrow r_{c}} \Gamma^{2}(r)=1 \Longrightarrow \lim _{r \rightarrow r_{c}} E(r)=0 .
$$

From the limiting behaviour of the functions in (2.23) at $r \rightarrow r_{c}$, we conclude that

$$
\lim _{r \rightarrow r_{c}} 2 E / \mathcal{M}^{2 / 3}=\lim _{r \rightarrow r_{c}}\left(\Gamma^{2}(r)-1\right) / \mathcal{M}^{2 / 3}=\text { const }
$$

and this constant may be zero. Note that with $\Gamma<0$ this means $\Gamma\left(r_{c}\right)=-1$. A central point where $\Gamma<0$ is the "second center of symmetry", in those models that have it. It is the antipodal point, in the spherical space, to the ordinary center. Having reached the antipodal point, we have added as much mass to the space as it can contain, and adding new mass is not possible. The condition $\Gamma\left(r_{c}\right)=-1$ must then be understood as follows: the active gravitational mass increases when we take away the rest mass from the object.

\section{$7 \quad$ Shell crossings in charged dust}

As is seen from (5.10) and (5.7), $F=0$ is equivalent to $R,_{r}=0$, so $F=0$ is a locus of shell crossing. Then, from (5.13) we see that $\Gamma F u^{R}$ must be negative for the density of dust to be positive. Since $u^{R}=\mathrm{d} R / \mathrm{d} s<0$ during collapse, $\Gamma F$ must then be positive.

We will investigate the occurrence of shell crossings in a configuration that avoids the Big Bang/Crunch singularities. Let us write the solution of (5.18) as follows

$$
F=I\left(\mathcal{M}, R, R_{1}\right)+g(\mathcal{M})
$$

where

$$
I\left(\mathcal{M}, R, R_{1}\right) \stackrel{\text { def }}{=}-\int_{R_{1}}^{R} \frac{1}{\left(u^{R}(\mathcal{M}, x)\right)^{3}}\left\{\Gamma, \mathcal{M}+\frac{1}{x \Gamma}\left[1-\frac{c^{4}}{G}\left(Q,,_{N}^{2}+Q Q,{ }_{N N}\right)\right]\right\} \mathrm{d} x,
$$

$R_{1}$ is the initial value of $R$ and $g(\mathcal{M})$ is an arbitrary function - the value of $F(\mathcal{M}, R)$ at $R=R_{1}$. In following the collapse of the central region forward in time, we eventually come close to the bounce point, where $R<R_{1}$. The integral becomes unbounded as $R$ approaches the bounce value (where $u^{R} \rightarrow 0$ ), and so, with $R<R_{1}$, the first term in (17.1) is positive when the expression in curly braces is negative.

We saw in Sec. 4 that there are dust particles with all values of $R$, including $R=0$. At the turning point $u^{R} \rightarrow 0$. The integrand is of the form $\left[\left(a x^{2}+b x+c\right)^{-3 / 2}\left(a_{2} x^{3}+a_{3} x^{2}\right)\right]$, and the trinomial has real zeros, so the integral is unbounded, which shows that $u^{R}=0$ is a coordinate singularity. $\Omega^{2}$ From (7.2) it is seen that as we get near to $\{R=0, \mathcal{M}=0\}$,

\footnotetext{
${ }^{2}$ Incidentally, the set where $\Gamma=0$ is also a coordinate singularity. This set is the "neck", known from studies of the Lemaitre-Tolman model, see Ref. [14.
} 
the coefficient $[1 /(x \Gamma)]$ will become unbounded. We know from the regularity conditions that $\lim _{r \rightarrow r_{c}} \Gamma^{2}=1, \lim _{r \rightarrow r_{c}} Q=0, \lim _{r \rightarrow r_{c}} Q,_{N}=$ const $<\infty$ and $\lim _{r \rightarrow r_{c}} Q / R=0$. Thus, as long as $Q,{ }_{N}{ }^{2}<G / c^{4}$, the term containing $1 /(x \Gamma)$ will dominate in the vicinity of $R=0$ and will determine the sign of the infinity in $F$. Now it turns out that the sign of $F$ will necessarily change to opposite during collapse: If $\Gamma>0$ and $g(\mathcal{M})>0$, then $F>0$ at $R=R_{1}$, but $F \rightarrow-\infty$ as $R \rightarrow 0$. If $\Gamma<0$, then $F<0$ can be achieved at $R=R_{1}$ by the choice $g(\mathcal{M})<0$, but $F \rightarrow+\infty$ as $R \rightarrow 0$. This means, there is necessarily a shell crossing somewhere at $R>0$ if $Q,{ }_{N}{ }^{2}<G / c^{4}$ holds all the way down to $\mathcal{M}=0$. This is the theorem proven by Ori [11, 12].

The infinity in $F$ could be avoided if the term in curly braces in (7.2) were zero at the same $x$, at which $u^{R}=0$. The zero of $u^{R}$ is given by (4.3), it is $R=R_{+}$. In that case $F$ is finite at $R=R_{+}$, and, by (5.13), $\epsilon$ becomes infinite, i.e. $R=R_{+}$becomes a true curvature singularity. Thus, also in this case, the charged dust cannot tunnel through the Reissner-Nordström throat.

The only situations in which both $\mathrm{BB} / \mathrm{BC}$ and shell crossing singularities could possibly be avoided are these:

1. When $\lim _{r \rightarrow r_{c}} Q,{ }_{N}^{2}=G / c^{4}$ - then, because of $\lim _{r \rightarrow r_{c}} Q / R=0$, the term $\Gamma, \mathcal{M}$ in (7.2) has a chance to outbalance the other one and secure the right sign of $F$ everywhere.

2. When $Q,{ }_{N}^{2}>G / c^{4}, E>0$ and $M<0$. Then, as seen from (4.3), both $R_{ \pm}>0$ and a nonsingular bounce at $R_{+}$, with no shell crossings, is possible.

Ori (private communication) found an example of a fully nonsingular bounce with $Q,{ }_{N}^{2}>G / c^{4}$, but has never published this result. An example of a nonsingular bounce with $Q,{ }_{N}^{2}<G / c^{4}$ will be given in Sec. 11.

\section{The evolution of charged dust in mass-curvature coordinates.}

In the mass-curvature coordinates, with $\Lambda=0$, it is easy to solve the evolution equation $\mathrm{d} R / \mathrm{d} s=u^{R}$. By this opportunity, we can again identify, by another method, all those solutions that avoid the $\mathrm{BB} / \mathrm{BC}$ singularity.

We take $\ell=+1$ for expansion and $\ell=-1$ for collapse 3 we denote

$$
2 E \stackrel{\text { def }}{=} \Gamma^{2}-1, \quad \Phi \stackrel{\text { def }}{=} \frac{Q^{2}\left(Q,,_{N}^{2}-G / c^{4}\right)}{2 E}
$$

and obtain from (5.8) with $\Lambda=0$

$$
\frac{\mathrm{d} R}{\mathrm{~d} s}=\ell \sqrt{2 E+2 M / R+2 E \Phi / R^{2}}
$$

The integral is different for each sign of $E$, and for $E \geq 0$ there are separate subcases depending on the values of $\Phi$ and $M ; \Phi<0$ corresponds to $Q,_{N}^{2}<G / c^{4}$. With $\Phi=0$ the solutions are of the same form as those in the $\mathrm{L}-\mathrm{T}$ model, although, if $Q \neq 0$, the effective mass $M$ still contains a contribution from the charges. With $E \neq 0$ it is most convenient to represent the solution $R(s)$ by parametric formulae $\{R(\omega), s(\omega)\}$.

\footnotetext{
${ }^{3}$ We refer here to the initial instant of evolution. In the nonsingular models, collapse will reverse into expansion during the evolution.
} 


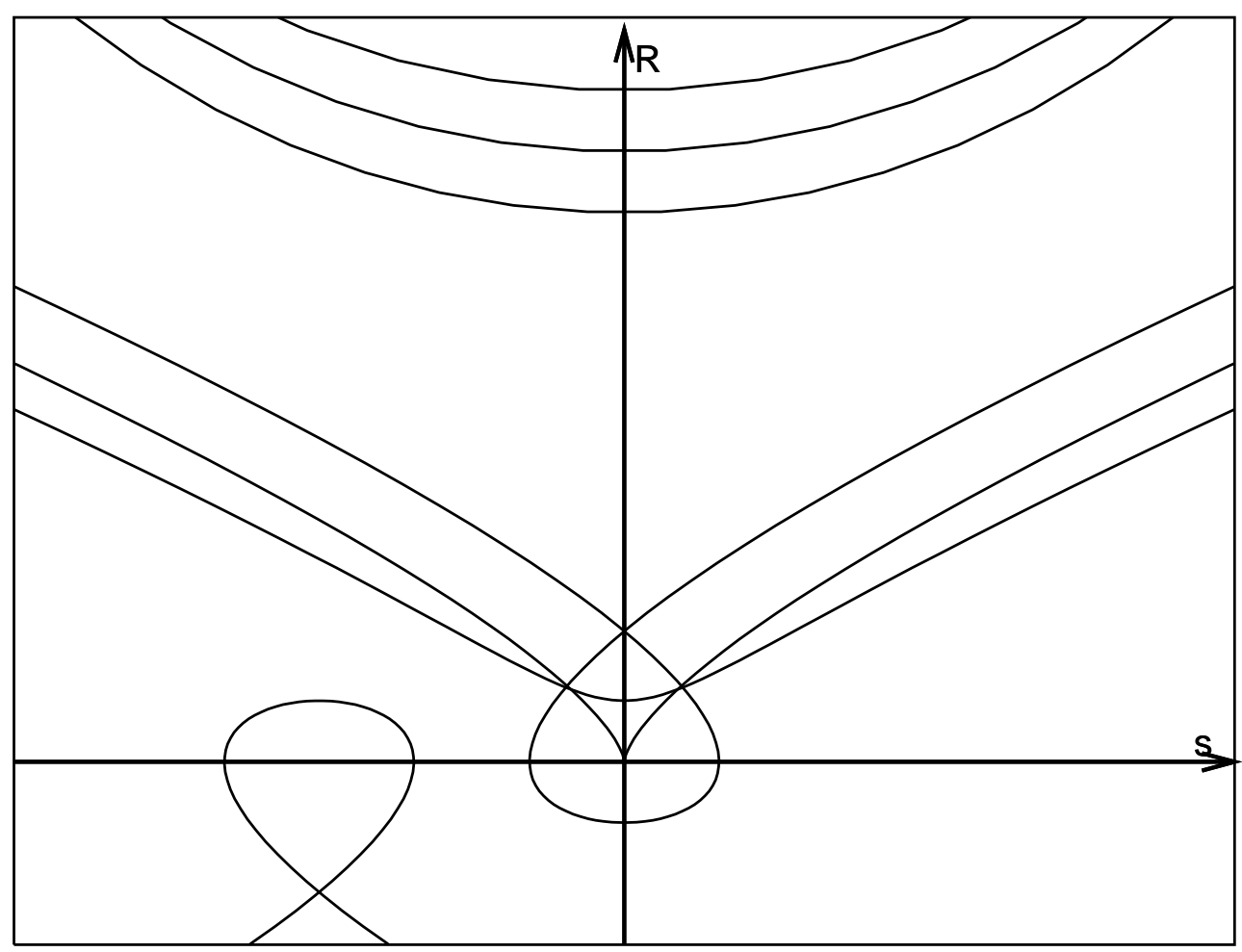

Figure 1: Schematic graphs of the solutions (8.3), with different values of $M$ and $\Phi$. Only those parts of the curves that lie above the $s$ axis describe physical situations. From bottom to top (counted at the left edge), the curves correspond to: 1. $\left\{\ell_{2}=-1, M<0, \Phi>0\right\}$. This model has a finite time of existence. 2. $\left\{\ell_{2}=+1, M>0, \Phi<0\right\}$. 3. $\left\{\ell_{2}=+1, M>0, \Phi=0\right\}$. This is the uncharged (Lemaitre Tolman) model, included for comparison. 4. $\left\{\ell_{2}=+1, M>0, \Phi>0\right\}$. The collapsing branch (left) ends in a singularity at $R=0$, the expanding branch (right) starts at the singularity at $R=0$. Models 1,3 and 4 are the only ones with singularities. 5. $\left\{\ell_{2}=+1, M<0, \Phi>0\right\}$. 6. $\left\{\ell_{2}=+1, M<0, \Phi=0\right\}$. 7 . $\left\{\ell_{2}=+1, M<0, \Phi<0\right\}$. The instant of time-symmetry is in general different on different $M$-shells. It was made the same in the figure only for better readability.

With $E>0$ and $\Phi<M^{2} /\left(4 E^{2}\right)$, the solution is

$$
\begin{aligned}
R & =\ell_{2} \sqrt{\frac{M^{2}}{4 E^{2}}-\Phi} \cosh \omega-\frac{M}{2 E}, \\
s-s_{B}(\mathcal{M}) & =\frac{\ell}{\sqrt{2 E}}\left(\sqrt{\frac{M^{2}}{4 E^{2}}-\Phi} \sinh \omega-\frac{M}{2 E} \omega\right),
\end{aligned}
$$

where $\omega$ is the parameter, $\ell_{2}= \pm 1$ and $s_{B}(\mathcal{M})$ is an arbitrary function of integration. In the $\mathrm{L}-\mathrm{T}$ limit $Q=0$, this function becomes the bang-time. In the bang-free models, $s_{B}(\mathcal{M})$ represents the instant at which $R$ achieves a minimum or a maximum.

The solution (8.3) has no BB/BC singularity when $\ell_{2}=+1$ and either $M<0$ or $\Phi<0$. Schematic graphs of the solutions are shown in Fig. 1.

With $E>0$ and $\Phi=M^{2} /\left(4 E^{2}\right)$, the solution is

$$
s-s_{B}(\mathcal{M})=\frac{\ell}{2 E}\left\{R-\frac{M}{2 E} \ln \left[\left(R+\frac{M}{2 E}\right) / R_{0}\right]\right\} .
$$

This one will have no $\mathrm{BB} / \mathrm{BC}$ singularity only if $M<0$. Schematic graphs of these solutions are shown in Fig. 2. 


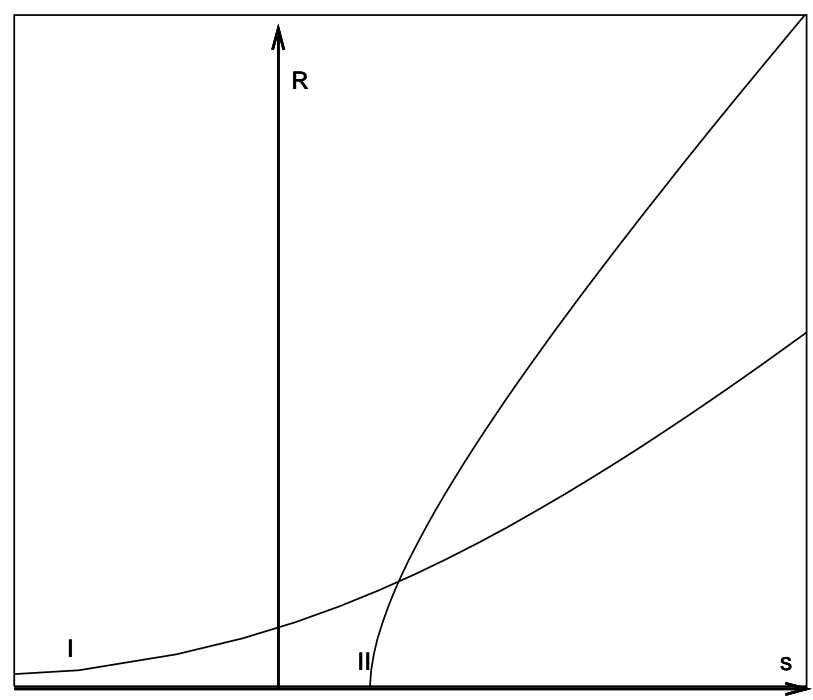

Figure 2: Schematic graphs of the solutions (8.4), with $\ell=+1$ and different values of $M$. Curve I is the nonsingular solution with $M<0$, curve II is a solution with $M>0$ and a singularity at finite $s$. In solution I $R$ tends to zero asymptotically as $s \rightarrow-\infty$.

With $E>0$ and $\Phi>M^{2} /\left(4 E^{2}\right)$, the solution is

$$
\begin{aligned}
R & =\sqrt{\Phi-\frac{M^{2}}{4 E^{2}}} \sinh \omega-\frac{M}{2 E} \\
s-s_{B}(\mathcal{M}) & =\frac{\ell}{\sqrt{2 E}}\left(\sqrt{\Phi-\frac{M^{2}}{4 E^{2}}} \cosh \omega-\frac{M}{2 E} \omega\right) .
\end{aligned}
$$

This model always runs into a BB/BC singularity at $\sinh \omega=M / \sqrt{4 E^{2} \Phi-M^{2}}$, independently of the sign of $M$. It has no uncharged limit $\Phi=0$. Schematic graphs of the solutions are shown in Fig. 3.

With $E=0$, we have to separately consider the subcase $M=0$. Unlike in neutral dust, this subcase is not vacuum (see (2.29)) ; it corresponds to the case when the electrostatic repulsion among the dust particles exactly balances the gravitational attraction, so that the effective mass is zero. This subcase exists with all signs of $E$, but does not require separate treatment when $E \neq 0$. (Such cases exist also in Newton's theory. The Newtonian solutions with $M=0$ and $E>0$ are expanding or collapsing with constant velocity, $R= \pm \sqrt{2 E}\left(t-t_{0}\right)+R_{0}$, those with $M=0=E$ are static.)

When $E=0$ and $M \neq 0$, the solution is

$$
s-s_{B}(\mathcal{M})=\frac{\ell}{3 M^{2}} \sqrt{2 M R+Q^{2}\left(Q,_{N}{ }^{2}-G / c^{4}\right)}\left[M R-Q^{2}\left(Q,,_{N}^{2}-G / c^{4}\right)\right],
$$

and it will not run into a $\mathrm{BB} / \mathrm{BC}$ singularity only if $M>0$ and $Q,,_{N}^{2}<G / c^{4}$. Schematic graphs of the solutions are shown in Fig. 4.

When $E=0=M$, the solution exists only when $Q,{ }_{N}{ }^{2}>G / c^{4}$, and it is

$$
R=\sqrt{2 \ell|Q| \sqrt{Q,,_{N}^{2}-G / c^{4}}\left[s-s_{B}(\mathcal{M})\right]}
$$




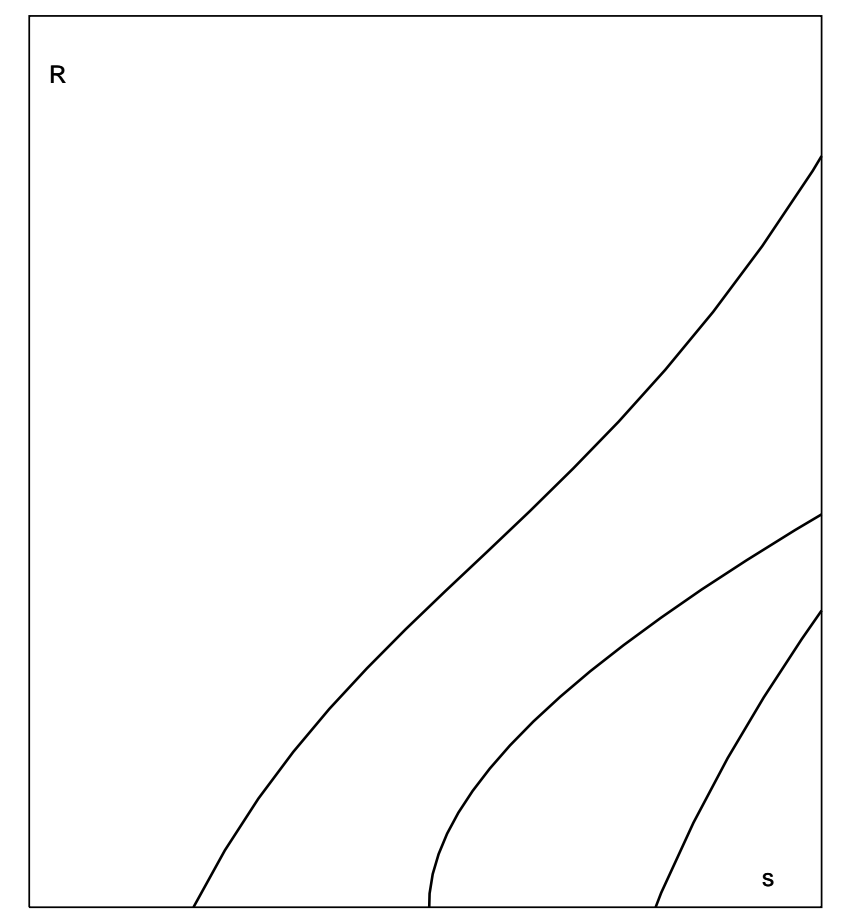

Figure 3: Schematic graphs of the solutions (8.5), with $\ell=+1$ and different values of $M$ and $\Phi$. Every solution has a singularity; only the slope of the curve at the singularity and the instant of the singularity depend on $M$ and $\Phi$.

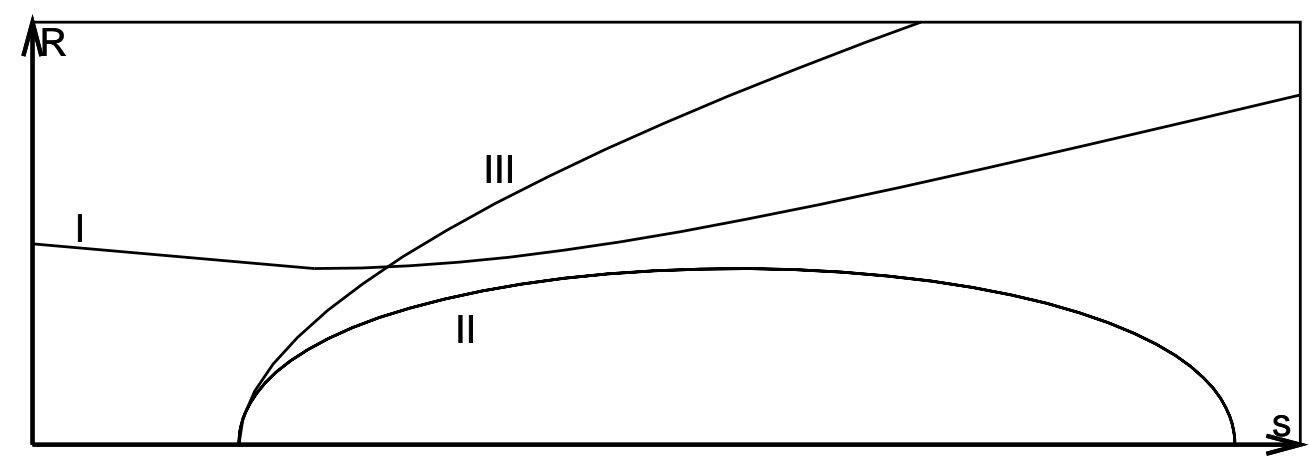

Figure 4: Schematic graphs of the solutions (8.6), with $\ell= \pm 1$ for curve I and $\ell=+1$ for the other two curves, and with different values of $M$ and $Q,{ }_{N}$. Curve $\mathrm{I}$ is the nonsingular solution with $M>0$ and $Q, N^{2}<G / c^{4}$, curve II corresponds to $M<0$ and $Q, N^{2}>G / c^{4}$, curve III corresponds to $M>0$ and $Q, N_{N}^{2}>G / c^{4}$. Model II achieves maximal expansion at $R=-Q^{2}\left(Q,{ }_{N}^{2}-G / c^{4}\right) /(2 M)$ and then recollapses. 


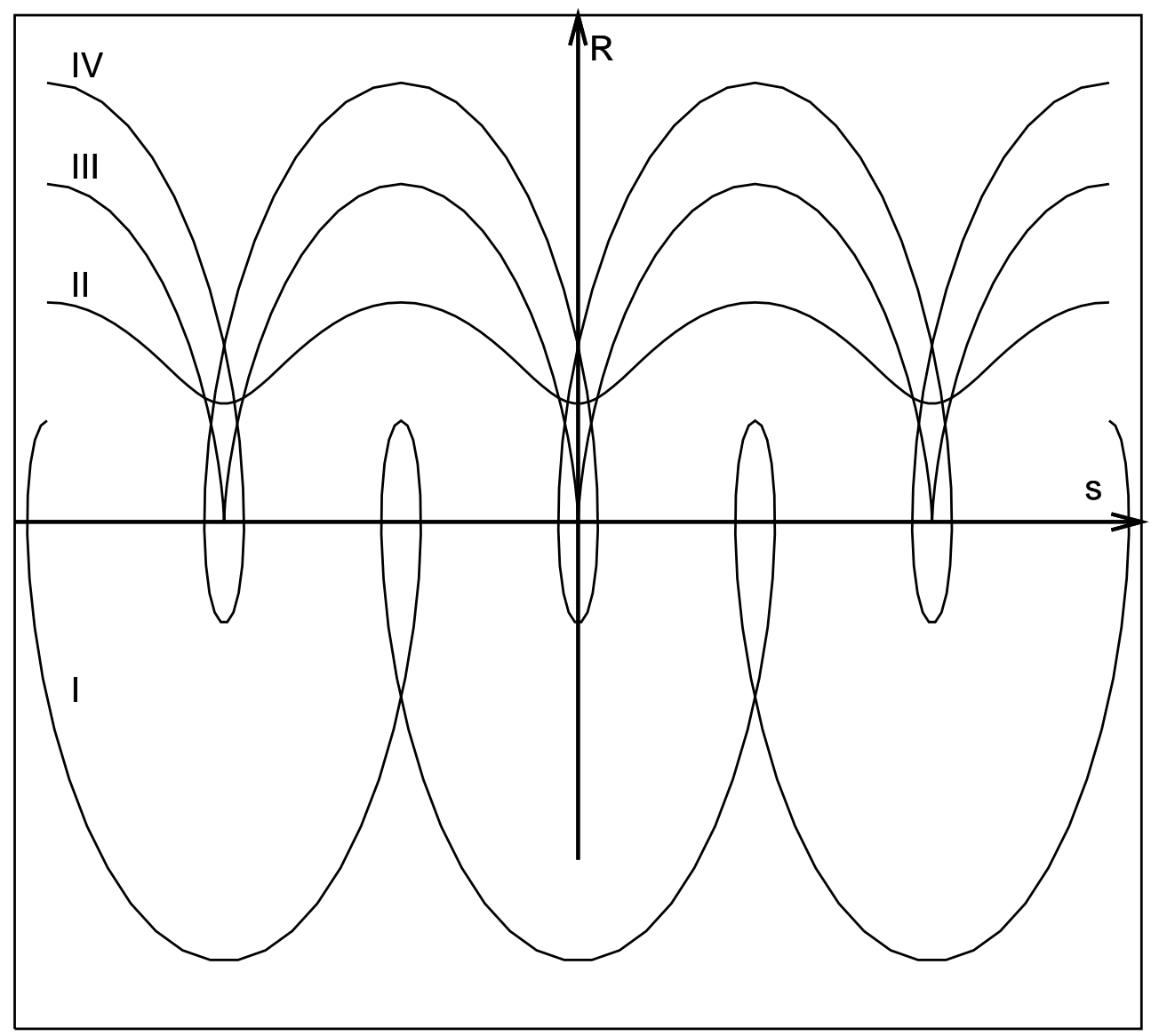

Figure 5: Schematic graphs of the solutions (8.8), with $\ell=+1$ and with different values of $M$ and $\Phi$. Only those parts of the curves that lie above the $s$-axis describe physical situations. The values of the parameters are as follows: Curve I $-M<0$ and $\Phi<0$; Curve II $-M>0$ and $\Phi>0$; Curve III $M>0$ and $\Phi=0$ (this is the Lemaitre - Tolman evolution, shown for comparison); Curve IV $-M>0$ and $\Phi<0$. Curve II is the nonsingular model, all the other models begin and end with a singularity, and the derivative $\mathrm{d} R / \mathrm{d} s$ is always infinite at the singularity. Different $M$-shells have different periods in $s$. The periods were made equal only to improve the readability of the picture.

This one always has a singularity. A plot has a similar shape as curve III in Fig. 4.

When $E<0$, a solution exists only with $\Phi<M^{2} /\left(4 E^{2}\right)$, and it is

$$
\begin{aligned}
R & =-\frac{M}{2 E}-\sqrt{\frac{M^{2}}{4 E^{2}}-\Phi} \cos \omega \\
s-s_{B}(\mathcal{M}) & =\frac{\ell}{\sqrt{-2 E}}\left(-\frac{M}{2 E} \omega-\sqrt{\frac{M^{2}}{4 E^{2}}-\Phi} \sin \omega\right) .
\end{aligned}
$$

This will avoid a $\mathrm{BB} / \mathrm{BC}$ singularity only if $M>0$ and $\Phi>0$. Schematic graphs of the solutions are shown in Fig. 5. This is a periodic solution, and the period is $T=$ $2 \pi M /(-2 E)^{3 / 2}$.

Note that if the functions $M(r), E(r)$ and $Q(r)$ are chosen in accordance with the regularity conditions of Sec. 6 , then the regular behaviour of $R$ at the center, $\lim _{r \rightarrow r_{c}} R / \mathcal{M}^{1 / 3}=$ $\beta(t)$, automatically follows in eqs. (8.3) - (8.6) and (8.8), but not in (8.7). 


\section{Transition from collapse to expansion by nonsingu- lar bounce}

We will now investigate transferring the solution (7.1) - (7.2) from the collapsing sector to the expanding sector. This is a nontrivial problem because, as we have seen, the bounce point along each worldline, where $u^{R}=0$, is a singularity of the mass-curvature coordinates, and we deduced (7.1) - (7.2) having $u^{R}<0$ in mind. On the other hand, all equations up to (5.18) apply independently of the sign of $u^{R}$.

Let us rewrite eq. (2.23) treating $(\mathcal{M}, R)$ as the independent variables and $t$ as the unknown function. Proceeding as in eqs. (5.6) - (5.7), we find that (2.23) is equivalent to

$$
\frac{\mathrm{d} t}{\mathrm{~d} R}= \pm \frac{\mathrm{e}^{-C(\mathcal{M}, R) / 2}}{\sqrt{\Gamma^{2}-1+2 M / R+Q^{2}\left(Q, N^{2}-G / c^{4}\right) / R^{2}-(1 / 3) \Lambda R^{2}}}
$$

where + corresponds to expansion, - to collapse, and $(\Gamma, M, Q)$ are now functions of $\mathcal{M}$. The solution of (9.1) is

$$
t= \pm J\left(\mathcal{M}, R, R_{0}\right)+t_{B}(\mathcal{M})
$$

where $t_{B}(\mathcal{M})$ is an arbitrary function and

$$
J\left(\mathcal{M}, R, R_{0}\right) \stackrel{\text { def }}{=} \int_{R_{0}}^{R} \frac{\mathrm{e}^{-C(\mathcal{M}, w) / 2} \mathrm{~d} w}{\sqrt{\Gamma^{2}-1+2 M / w+Q^{2}\left(Q,,^{2}-G / c^{4}\right) / w^{2}-(1 / 3) \Lambda w^{2}}}
$$

The constant $R_{0}$ is an arbitrarily chosen initial point of integration, and $t_{B}(\mathcal{M})$ is the integration "constant", an analogue of the Lemaitre - Tolman bang-time function. Here,

$t_{B}(\mathcal{M})=\left.t\right|_{R=R_{0}}$. Since $R$ is the only quantity that may depend on $t$ in the comoving coordinates, we see that it depends on $t$ always through the combination $\left(t-t_{B}\right)$.

Note that eqs. (9.1) - (9.3) apply only throughout a single expansion phase or a single collapse phase. Thus, when we want to consider the transfer from collapse to expansion in those models in which it is nonsingular, we have to choose $R_{0}=R_{\min }$, the minimal value achieved by $R$ for a given $\mathcal{M}$, and thus $t=t_{B}$ will correspond to the bounce instant.

Consider now a pair of solutions (9.2) - (9.3), the first one collapsing, the other one expanding, both with the same function $t_{B}(\mathcal{M})$, and consider the two solutions at the same values of $\left(\mathcal{M}, R, R_{0}\right)$. In accordance with the previous paragraph, we have to choose $t_{B}(\mathcal{M})=t\left(\mathcal{M}, R_{\text {min }}\right)$ for both. Let the collapsing solution go through these values of $\left(\mathcal{M}, R, R_{0}\right)$ at $t=t_{c}$, and the expanding one at $t=t_{e}$. We have $t_{c}=-J\left(\mathcal{M}, R, R_{0}\right)+t_{B}$ for the collapsing solution, and $t_{e}=+J\left(\mathcal{M}, R, R_{0}\right)+t_{B}$ for the expanding one. Thus, $t_{c}+t_{e}=2 t_{B}$, i.e. $t_{B}(\mathcal{M})$ is the midpoint of the interval $\left[t_{c}, t_{e}\right]$. In general, it thus depends on $\mathcal{M}$, i.e. is different for each mass-shell. The case $t_{B}=$ const, i.e. when the midpoint has the same value for all masses, corresponds to the evolution that is time-symmetric with respect to $t=t_{B}$.

Now consider eq. (5.10) (recall: the $f$ in (5.10) is the comoving time $t$ ). Let us consider a pair of curves $\left(C_{c}, C_{e}\right)$ in the $(R, \mathcal{M})$ surface given by the same equation $R=R_{c e}(\mathcal{M})$, one curve in the collapsing region, the other one in the expanding region. Write eq. (5.10) first for the collapsing solution, along $C_{c}$ :

$$
\frac{\mathrm{e}^{C / 2}}{\Gamma-Q Q,_{N} / R}\left[-J,_{\mathcal{M}}\left(\mathcal{M}, R, R_{0}\right)+t_{B, \mathcal{M}}(\mathcal{M})\right]=I_{c}\left(\mathcal{M}, R, R_{1}\right)+g_{c}(\mathcal{M}),
$$


where the functions $I$ and $g$ are those defined in (7.1) - (7.2), and the subscript $c$ refers to collapse. Write the same equation for the expanding solution, along $C_{e}$ :

$$
\frac{\mathrm{e}^{C / 2}}{\Gamma-Q Q,_{N} / R}\left[+J,_{\mathcal{M}}\left(\mathcal{M}, R, R_{0}\right)+t_{B, \mathcal{M}}(\mathcal{M})\right]=I_{e}\left(\mathcal{M}, R, R_{1}\right)+g_{e}(\mathcal{M}) .
$$

The $I_{e}$ in (9.5) differs from the $I_{c}$ in (9.4) only by the sign of $u^{R}$, otherwise all quantities in $I_{e}$ are the same as their counterparts in $I_{c}$. Thus, along the chosen pair $\left(C_{c}, C_{e}\right)$, we have $I_{c}+I_{e}=0$. Knowing this, and adding eqs. (9.4) and (9.5), we obtain

$$
\frac{\mathrm{e}^{C / 2}}{\Gamma-Q Q,_{N} / R} 2 t_{B, \mathcal{M}}(\mathcal{M})=g_{c}(\mathcal{M})+g_{e}(\mathcal{M}) .
$$

This is the equation that relates $g(\mathcal{M})$ for the collapsing solution in (7.1) - (7.2) to the corresponding function in the expanding solution. For a time-symmetric evolution, and only then, we thus get $g_{e}=-g_{c}$. Note that if the l.h.s. of (9.6) is positive and large, while $g_{c}$ is positive and smaller than the l.h.s., then $g_{e}$ cannot be negative. This observation will prove important below, in analysing the existence/avoidance of shell crossings.

We recall (see the comment after (5.13) ) that $F>0$ where $\Gamma u^{R}<0$ and $F<0$ where $\Gamma u^{R}>0$. As the bounce point is approached from the $u^{R}>0$ sector in the central region, $R$ eventually becomes smaller than $R_{1}, F$ becomes unbounded, and it must tend to $-\infty$. We concluded before that the expression in curly braces must be negative if shell crossings are to be avoided in collapse. Thus, the first term in (7.1) - (7.2) gives the correct behaviour of $F$ in approaching the bounce point from the $u^{R}>0$ sector.

The function $F$ is thus discontinuous at the bounce set, it tends to $+\infty$ when this set is approached from the $u^{R}<0$ sector, and to $-\infty$ when it is approached from the $u^{R}>0$ sector. But, as seen from eqs. (5.11), the metric components depend on $F^{2}, F / u^{R}$ and $\left(u^{R}\right)^{2}$, and so they do not jump from one infinity to the other. Then, since $F u^{R}$ is finite at bounce and does not change sign, we see from (5.13) that the mass density is continuous across the bounce set.

Let us note that only the integral term $I$ in (7.1) simply changes sign when we go over from collapse to expansion. The function $g(\mathcal{M})$ transforms by (9.6), and it changes to its negative only for time-symmetric evolution. Thus, if we have guaranteed that there are no shell crossings before the bounce by choosing such $g$ that gives $I+g>0$ in the collapse phase, but the evolution is not time-symmetric $\left(t_{B, \mathcal{M}} \neq 0\right)$, then $I+g<0$ will in general fail to hold in the expansion phase, and shell crossings will appear after the bounce. We shall come back to this question later.

\section{Nonsingular bounce of a weakly charged dust}

From now on we assume $Q,,_{N}^{2}<G / c^{4}$ (weakly charged dust).

The cases $E \geq 0$ and $E<0$ have to be considered separately. In the first case, if a totally nonsingular model existed, it would be first collapsing from an arbitrary initial density, then it would go through just one bounce, and re-expand so that each non-central shell achieves an infinite radius. In the second case, each shell would oscillate between the two turning values of $R$. At each turning value, $u^{R}=0$ and $|F| \rightarrow \infty$, and we have to 
choose the arbitrary functions in (77.1) - (7.2) so that the infinity has always the right sign (e.g. $F \rightarrow+\infty$ when $u^{R}<0$ and $\Gamma>0$ before the turning point). Then, in principle we can secure the right sign in two ways in each case: by imposing conditions on $\Gamma, \mathcal{M}$ or on

$$
F_{1} \stackrel{\text { def }}{=} 1-\left(c^{4} / G\right)\left(Q,,^{2}+Q Q, N N\right)
$$

depending on which one dominates in a given situation.

We first observe that a weakly charged nonsingular dust distribution with $E \geq 0$ cannot exist. We assume that the regularity conditions at the center hold, and that $Q,{ }_{N}^{2} \underset{\mathcal{M} \rightarrow 0}{\longrightarrow} G / c^{4}$. Throughout the proof, and then in deducing the conditions for no shell crossings in an $E<0$ model, we assume $u^{R}<0$ (collapse), just for definiteness. In the end, it will be easy to see that the same conditions must apply in the expansion phase.

Suppose, for the beginning, that $E=0$ and consider collapse. Then $\Gamma=\sqrt{2 E+1}= \pm 1$ is constant throughout the spacetime, and $\Gamma, \mathcal{M} \equiv 0$. Thus, $F_{1}$ must determine the sign of $F$ at the bounce point. To secure the right sign also in the central region it must obey, in a vicinity of the center, $F_{1}<0$, independently of the sign of $\Gamma$ (because $\Gamma F$ must have the same sign as long as $u^{R}$ does not change sign). In a vicinity of the center the quantity $\left(1-c^{4} Q, N^{2} / G\right)$ is positive by assumption, while $Q,{ }_{N}^{2}=G / c^{4}$ at the center. We have to consider two cases:

1. Assume $Q,,_{N}=+\sqrt{G} / c^{2}$ at the center. This means that $Q,,_{N}>0$ in some vicinity of the center. Since, by the regularity conditions, $Q=0$ at the center, we have $Q>0$ around the center. Then the condition $F_{1}<0$ means $Q,{ }_{N N}>\left(G / c^{4}-Q,,_{N}^{2}\right) / Q>0$, which implies that $Q,{ }_{N}$ in the vicinity of $\mathcal{M}=0$ is larger than at $\mathcal{M}=0$, i.e. larger than $\sqrt{G} / c^{2}$. This is a contradiction with the assumption.

2. Assume $Q,_{N}=-\sqrt{G} / c^{2}$ at the center. Then $Q,_{N}<0$ in some vicinity of the center, and from the regularity conditions $Q<0$ in the same vicinity. Then $F_{1}<0$ implies $Q,{ }_{N N}<\left(G / c^{4}-Q,_{N}^{2}\right) / Q<0$, which means $Q,,_{N}<-\sqrt{G} / c^{2}$ in a neighbourhood of the center, in contradiction to the assumption.

Thus, $E=0$ leads to a contradiction and is thereby excluded.

Suppose then that $E>0$ and that $F_{1} /(R \Gamma)$ becomes negligible as $x \rightarrow 0$, so that the sign of $\Gamma, \mathcal{M}$ determines the sign of infinity of $F$ at bounce. We still consider collapse. Since, with $\Gamma>0$ and $u^{R}<0, F$ must be positive, $\Gamma, \mathcal{M}<0$ is the right choice. The regularity conditions require that $\Gamma^{2}=1$ at the center. If $\Gamma>0$, then $\Gamma=+1$ at the center, and then $\Gamma, \mathcal{M}<0$ means $0<\Gamma<1$ in the vicinity of the center. But $2 E=\Gamma^{2}-1$, so this implies $E<0$. (With $\Gamma<0$, the argument is similar: then $F<0$, so $\Gamma, \mathcal{M}>0$ and $\Gamma=-1$ at the center, which implies $E<0$ in the neighbourhood.)

Then suppose that $E>0$ and that the limit at $\mathcal{M} \rightarrow 0$ of $F_{1} / R$ is finite (which means that the limit of $F_{1} / \mathcal{M}^{1 / 3}$ must be finite). As $R \rightarrow \infty$, the contribution from $F_{1} /(x \Gamma)$ in (7.2) becomes negligible, and $R>R_{1}$ eventually. Thus, with $\Gamma>0, F$ must go to $+\infty$ as $R \rightarrow \infty$, so $\Gamma, \mathcal{M}>0$. In the vicinity of the center, where $R<R_{1}$, we must have $\Gamma, \mathcal{M}+F_{1} /(R \Gamma)<0$, so $F_{1}<0$. The condition $F_{1}<0$ also follows when $\Gamma<0$. Thus $F_{1} / \mathcal{M}^{1 / 3}<0$ at the center and, by continuity, in some neighbourhood of the center. Then we use the same argument as above to show that it leads to a contradiction in every case.

In the case $E<0$, these problems can be avoided. With $E<0$, there are two turning points, at $R=R_{+}$and at $R=R_{-}>R_{+}$. The integral in (7.2) becomes unbounded at both $R_{+}$and $R_{-}$. The signs of the two infinities can then be imposed independently. The 
argument used after (10.1) still applies, so $F_{1}$ cannot secure the correct sign at $R_{+}$. Thus we have to assume that the limit of $F_{1} / R$ as $\mathcal{M} \rightarrow 0$ is zero, so that $\Gamma, \mathcal{M}<0$ secures the right behaviour at the inner turning point. Then we require that at $R_{-}$the inequality $1-\left(c^{4} / G\right)\left(Q,_{N}^{2}+Q Q,,_{N}\right)>-\Gamma \Gamma, \mathcal{M}_{-}$holds, which reads explicitly:

$$
1-\frac{c^{4}}{G}\left(Q,,_{N}^{2}+Q Q, N N\right)>\frac{\Gamma \Gamma, \mathcal{M}}{2 E}\left[M+\sqrt{M^{2}-2 E Q^{2}\left(Q,,_{N}^{2}-G / c^{4}\right)}\right] .
$$

Since $\Gamma, \mathcal{M}<0$ and $E<0$ in a neighbourhood of $\mathcal{M}=0$, this implies the following necessary condition;

$$
1-\frac{c^{4}}{G}\left(Q,,^{2}+Q Q, N N\right)>\frac{\Gamma \Gamma, \mathcal{M}}{2 E} M
$$

Since the integral in (7.2) goes to $+\infty$ at both turning points, it must have at least one local minimum somewhere in $\left(R_{+}, R_{-}\right)$. If $-\infty<I_{\min }<0$ at the smallest of these minima, then this can be corrected by the choice of $g(\mathcal{M})$. Also, $I$ equals to zero in at least one point - at $R=R_{1}$, where $R_{+}<R_{1}<R_{-}$. Thus, $I$ cannot be positive everywhere, so necessarily $g(\mathcal{M})>0$ to avoid $F=0$ anywhere.

In summary, a weakly charged configuration that could bounce singularity-free through the $\mathrm{R}-\mathrm{N}$ wormhole must obey the following necessary conditions in a neighbourhood of the center:

(1) $E<0$ (as $E \geq 0$ was eliminated above);

(2) $E \geq-1 / 2$ (since $\Gamma$ is the primary arbitrary function, and $E$ was defined as an auxiliary quantity by $\left.2 E=\Gamma^{2}-1\right)$ t;

(3) $\lim _{r \rightarrow r_{c}} F_{1} / \mathcal{M}^{1 / 3}=0$, in consequence of $\lim _{r \rightarrow r_{c}} F_{1} / R=0$ and of (6.2) (recall that $\beta \neq 0$ in $(\underline{6.2})$.

(4) $\Gamma, \mathcal{M}<0$ (to secure the right sign of $F$ at the inner turning point);

(5) $Q,,_{N}^{2}<G / c^{4}$ at $N>0$ and $Q,,_{N}^{2}=G / c^{4}$ at $N=0$ (the defining condition for weakly charged dust);

(6) $M \equiv \mathcal{M}-Q Q,_{N} \Gamma>0$ (a necessary condition for no $\mathrm{BB} / \mathrm{BC}$ with $E<0$, as shown in Secs. 4 and 8$)$;

(7) $M^{2}-2 E Q^{2}\left(Q,{ }_{N}{ }^{2}-G / c^{4}\right)>0$ (a necessary condition for the existence of a solution of (2.23), see (4.2); equality leads to a static solution);

(8) condition (10.3) (a necessary condition for (10.2) to be obeyed);

(9) condition (10.2) (to secure the right sign of $F$ at the outer turning point). In addition to that, all the regularity conditions of sec. 6 must be obeyed.

Conditions (1) - (9) must hold in a neighbourhood of the center. At the center, the left-hand sides of conditions $(1)$ and $(6-9)$ must have zero limits.

Condition (10.2) should hold at the state of maximal expansion. However, for those shells that contain small mass (are sufficiently close to the center), the value of $R_{-}$will be arbitrarily small. Thus (10.2) must in fact hold down to arbitrarily small values of $\mathcal{M}$, with only $\mathcal{M}=0$ being excluded, where both sides of (10.2) should be zero.

Now, a glance at eqs. (7.1) - (7.2) suffices to see that the same conditions (1) - (9) must apply in the expansion phase, where $u^{R}>0$ and $\Gamma F<0$, if shell crossings are to

\footnotetext{
${ }^{4}$ The condition $E \geq-1 / 2$ finds a clearer explanation in the Lemaittre - Tolman (zero charge) limit, where it follows from the requirement of the right signature. It is also equivalent to the statement that there can be no turning points for radial motion of uncharged dust inside the apparent horizon.
} 
be avoided. The two functions $g$ are related to each other by (9.6), and it may happen that if we guaranteed $F=I_{c}+g_{c}>0$ in collapse, then $I_{e}+g_{e}=-I_{c}+g_{e}$ will refuse to be negative in some range during expansion, thus indicating a shell crossing. But if the bounce is time-symmetric, so that $g_{e}=-g_{c}$, then, having guaranteed no shell crossings in collapse, we know that there will be no shell crossings in the subsequent expansion phase. However, the next bounce will in general no longer be time-symmetric, and shell crossings will appear in later cycles. The shell crossings might be avoided for ever only if all bounces were time-symmetric. Equation (8.8) implies that such models might exist: the period of oscillations $T=2 \pi M /(-2 E)^{3 / 2}$ will be independent of $\mathcal{M}$ when $M /(-2 E)^{3 / 2}$ is constant. Together with $t_{B}$ being constant, such a condition reduces the Lemaitre - Tolman model to the Friedmann limit [5], but here we still have the function $Q$ that generates the electric field. With $T=T_{0}=$ const, the function $Q(N)$, in general arbitrary, has to obey

$$
Q Q,_{N}=\frac{G}{c^{4} \mathcal{M},_{N}}\left[\mathcal{M}-\frac{T_{0}}{2 \pi}\left(1-\Gamma^{2}\right)^{3 / 2}\right],
$$

and it can still obey the regularity conditions $Q=0$ and $\left|Q,_{N}\right|=\sqrt{G} / c^{2}$ at the center, if $\mathcal{M}$ and $\Gamma$ obey their regularity conditions. Also, (10.4) does not lead to any simple contradiction with conditions $(5-9)$. An explicit example of a configuration obeying (10.4) (or a proof that it does not exist) remains to be found.

Equation (10.4) is a consequence of the requirement that the period with respect to the proper time $s$ is independent of $\mathcal{M}$. It is easy to find from (9.1) that the period with respect to the time coordinate $t$ (equal to twice the integral of (9.1) from $R_{\text {min }}=R_{+}$to $\left.R_{\text {max }}=R_{-}\right)$is $2 \pi \mathrm{e}^{-C(\mathcal{M}, \bar{R}(\mathcal{M})) / 2} M /(-2 E)^{3 / 2}$, where $\bar{R}(\mathcal{M})$ is an intermediate value of $R$ between $R_{\min }$ and $R_{\max }$ (from the mean value theorem for integrals). Thus, the constancy of the period in $t$ also imposes an additional equation on $Q$. Here, however, an example could be found only numerically.

It should be noted that $E$ need not have the same sign in the whole volume of the charged dust. However, the change of sign of $E$ will have no influence on the conditions stated above. If it gets positive in a vicinity of the center, then nonsingular bounce cannot occur, as shown above. If it gets negative in a vicinity of the center, then it is negative in an open range of values of $\mathcal{M}$. Then, within the same open set there will be outer turning points of some mass-shells, so (10.3) and (10.2) must anyway hold close to the center. However, if $E$ becomes positive at some $\mathcal{M}=\mathcal{M}_{0}$, then there are no outer turning points at $\mathcal{M} \geq \mathcal{M}_{0}$, and the inequalities (10.2) and (10.3) need not be imposed in that region.

We have not yet excluded one more pathological situation that could occur with $E<0$. The arbitrary functions may be chosen so that the period of oscillations tends to zero as $\mathcal{M} \rightarrow 0$, as in Fig. 6. The thicker curve (call it $E_{\mathcal{M}}$ ) goes through the minima of the evolution curves $R(t, \mathcal{M})$, each of them corresponding to a fixed value of $\mathcal{M}$. In the figure, the slope $\alpha(\mathcal{M})$ of $E_{\mathcal{M}}$ is at each point smaller than the slope of the evolution curve that passes through the same point, and the shell crossings are inevitable. We could try to avoid it by requiring that $\alpha(\mathcal{M})$ is sufficiently large, but this would not work: the period of a curve with larger $\mathcal{M}$ would be larger than the period of a curve with smaller $\mathcal{M}$, and an intersection would be sure to occur after several cycles. With the period tending to zero as $\mathcal{M} \rightarrow 0$, the neighbourhood of the center $\mathcal{M}=0$ would be densely filled with shell crossings. Thus, the only way to avoid such a pathology is to choose the arbitrary functions so that the period tends to a nonzero value as $\mathcal{M} \rightarrow 0$. 


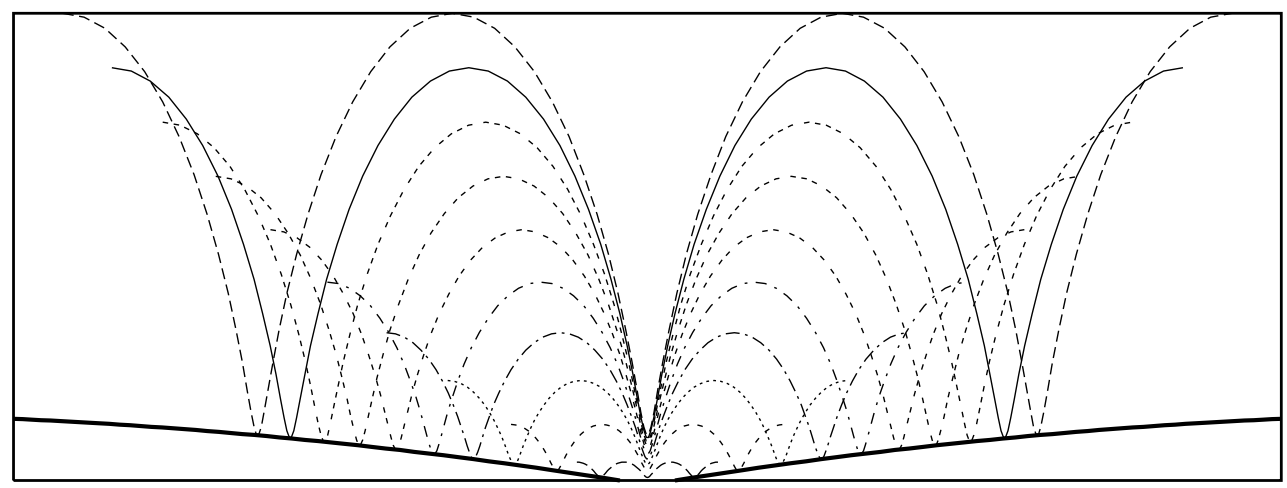

Figure 6: A possible pathology of an oscillating model with $E<0$ : the period of oscillations tends to zero as the center of symmetry is approached (the curves are graphs of the functions $R(t, \mathcal{M})$ for different values of $\mathcal{M}$ ). The thicker curve goes through the minima of the evolution graphs. (The model shown in the figure is time-symmetric with respect to the bounce in the middle of the axis.)

Equation (8.8) will help us in choosing the right functions. We assume that nonzero intervals of proper time along each worldline are mapped into nonzero intervals of the comoving time-coordinate and vice versa (i.e. that the metric function $\mathrm{e}^{C}$ in does not tend to zero or to infinity at the axis $\mathcal{M}=0$ ). Thus, if we choose the functions so that the period in $s$ in eq. (8.8) is nonzero, then the period in $t$ will also be nonzero. Note then that if $E$ obeys (6.4) with a nonzero constant, then, in consequence of condition (5), the period of $R$ in $s$ will tend to zero as $\mathcal{M} \rightarrow 0$. To avoid this, $E$ must tend to zero at the center faster than $\mathcal{M}^{2 / 3}$.

\section{An example}

In order to prove that conditions (1) - (9) from the previous section are not mutually contradictory, we shall now provide an example of a model that obeys them. It is simply the first model that has successfully passed all the tests, and we do not claim that it is physically important or realistic - it is only meant to be a proof of existence.

Since the functions appearing in the inequalities are rather complicated, the proof that the inequalities are all obeyed will be given mainly by numerical graphs. The connection between $\Gamma, N$ and $\mathcal{M}$ provided by (5.16) causes that even quite simple functions $E(N)$ that obey the regularity conditions lead to complicated expressions for $\mathcal{M}(N)$. However, we will verify by exact methods that in a vicinity of the center and at infinity the functions indeed behave in the same way as the graphs indicate.

Choose, in agreement with the regularity conditions and the weak-charge conditions:

$$
Q(N)=q \frac{\sqrt{G} N_{0}}{c^{2}} p(x),
$$


where $q= \pm 1$, to allow for any sign of the charge, $x \stackrel{\text { def }}{=} N / N_{0}, N_{0}$ is a constant, and

$$
p(x)=x /(1+x)^{2} .
$$

Then

$$
Q,_{N}=q \frac{\sqrt{G}}{c^{2}} \frac{1-x}{(1+x)^{3}} .
$$

This charge is zero at $x=0$, its absolute value increases at first, but then decreases and tends to zero as $x \rightarrow \infty$. Thus $Q, N$, which is proportional to the charge density (see $(2.20)$ ), changes sign at $x=1$, but it obeys condition (5) everywhere.

Further, we have

$$
F_{1}(x) \stackrel{\text { def }}{=} 1-\frac{c^{4}}{G}\left(Q,,^{2}+Q Q, N N\right)=1-\frac{3 x^{2}-6 x+1}{(1+x)^{6}} .
$$

Fig. 7 shows the shape of the functions $p(x), \mathrm{d} p / \mathrm{d} x$ and $F_{1}(x)$ (for all these functions it can be easily verified that the graphs show their behaviour faithfully, with no important details being hidden beyond the range of the graph or in a small vicinity of zero).

We choose now the function $E(N)$ so that the period $2 \pi M /(-2 E)^{3 / 2}$ in (8.8) has a nonzero limit at $N \rightarrow 0$. In order to keep $-1 / 2 \leq E<0$ in the whole range, and to avoid $\Gamma$ and $\mathcal{M}=\left(G / c^{4}\right) \int(1 / \Gamma) \mathrm{d} N$ being too complicated, we choose the trial form $2 E=-b x^{a} /\left(1+b x^{a}\right)$, with $a, b$ being constant, and we find from (8.8) that the period will have a nonzero (actually, infinite) limit at $N=0$ if $a=5 / 3$. This will also guarantee, via (8.8), that the limit of $R / \mathcal{M}^{1 / 3}$ at $x \rightarrow 0$ will be a nonzero function independent of $\mathcal{M}$, just as (6.2) requires. Thus

$$
2 E=-\frac{b x^{5 / 3}}{1+b x^{5 / 3}}
$$

With such $E$, the limiting period of oscillations in (8.8) is infinite, and

$$
\Gamma(x)=\frac{1}{\sqrt{1+b x^{5 / 3}}}
$$

which obeys condition (4), and, further:

$$
\mathcal{M}(x)=\frac{G N_{0}}{c^{4}} \int_{0}^{x} \frac{\mathrm{d} x^{\prime}}{\sqrt{1+b x^{\prime 5 / 3}}} \stackrel{\text { def }}{=} \frac{G N_{0}}{c^{4}} \mu(x) .
$$

The $\mu(x)$ is an increasing function for all $x>0$; its graph would almost coincide with the graph of $F_{2}$ shown in Fig. 7, but see the inset (actually, $\mu(x)>F_{2}(x)$ for $0<x<1$, the two curves intersect at $x=1$, and for $\left.x>1 \mu(x)<F_{2}(x)\right)$. Note that such $\mathcal{M}$ obeys $\lim _{x \rightarrow 0} \mathcal{M} / x=G N_{0} / c^{4} \neq 0$, while $\lim _{x \rightarrow 0} F_{1} / x=12$, so condition (3) is obeyed. We have now:

$$
\begin{gathered}
M \quad \\
F_{2}(x) \stackrel{\mathcal{M}-Q Q,_{N} \Gamma=\frac{G N_{0}}{c^{4}} F_{2}(x),}{\stackrel{\text { def }}{=}} \mu(x)-\frac{x(1-x)}{(1+x)^{5} \sqrt{1+b x^{5 / 3}}} .
\end{gathered}
$$




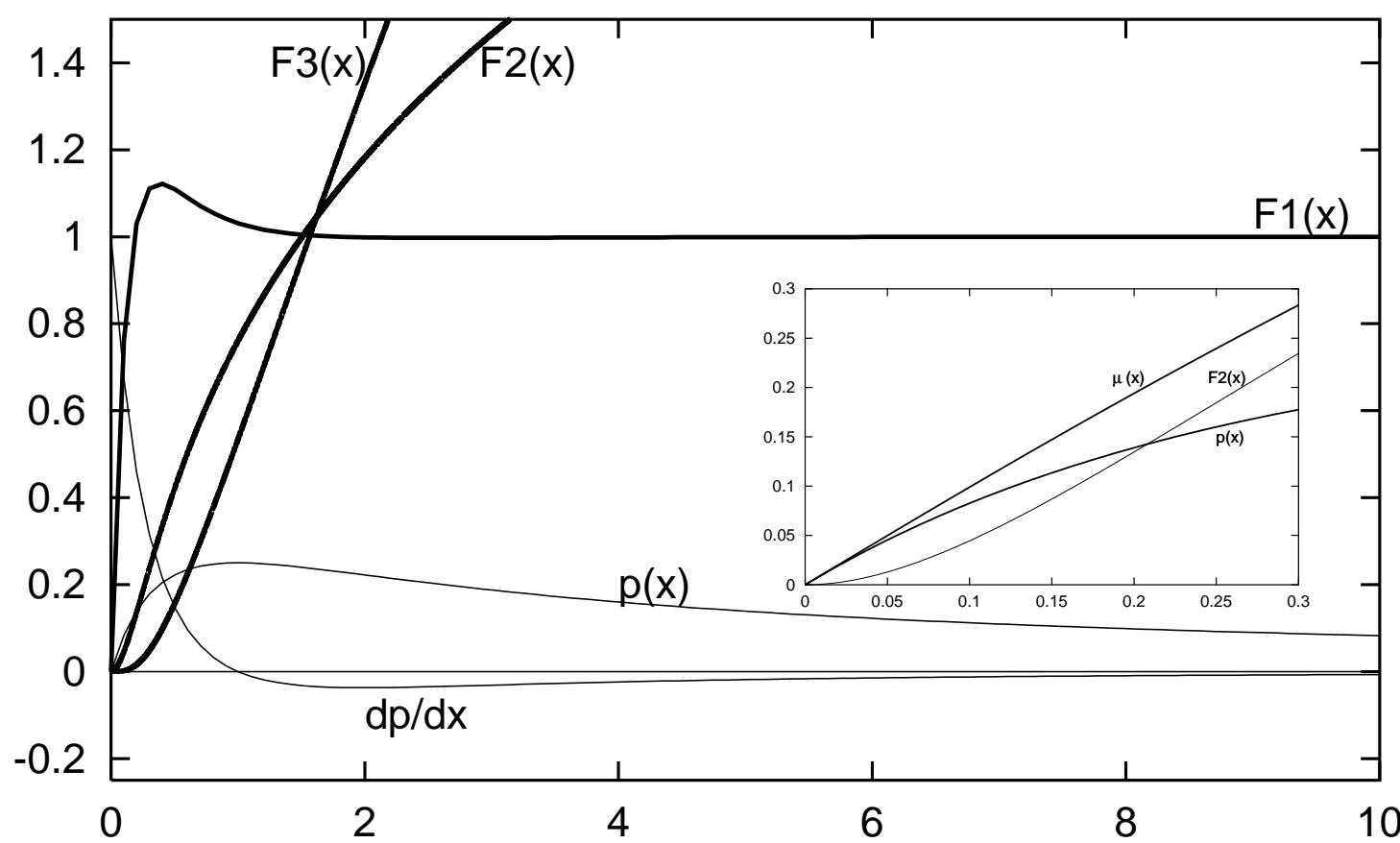

Figure 7: Graphs of the functions $p(x), \mathrm{d} p / \mathrm{d} x, F_{1}(x), F_{2}(x)$ and $F_{3}(x)$. Note that $-1<\mathrm{d} p / \mathrm{d} x<+1$ and $F_{2}(x)>0, F_{3}(x)>0$ for all $x>0$, thus conditions (5), (6) and (7) are fulfilled. All the functions are zero at $x=0$. As $x \rightarrow \infty, F_{2}$ and $F_{3}$ tend to $\infty, F_{1}$ tends asymptotically to 1 , while $p$ and $\mathrm{d} p / \mathrm{d} x$ tend asymptotically to 0 . The inset shows the functions $p(x), F_{2}(x)$ and $\mu(x)$ in a vicinity of $x=0$. It shows that, with our chosen value $b=2.5, \mu(x)>p(x)$ for $x>0$.

It is easy to verify that $F_{2}>0$ for all $x>0,5$ so condition (6) is fulfilled. The graph of $F_{2}$ is shown in Fig. 7 with $b=2.5$ (why this value - see below).

Condition (7) is equivalent to

$$
F_{3}(x)>0, \quad F_{3}(x) \stackrel{\text { def }}{=} F_{2}^{2}(x)-F_{6}(x),
$$

where

$$
F_{6}(x) \stackrel{\text { def }}{=}-2 E p^{2}\left(1-p_{x}^{2}\right)=\frac{b x^{11 / 3}}{\left(1+b x^{5 / 3}\right)(1+x)^{4}}\left[1-\frac{(1-x)^{2}}{(1+x)^{6}}\right] .
$$

The graph of $F_{3}(x)$ with $b=2.5$ is shown in Fig. 7. Since we have verified that $M \geq 0$, $E<0$ and $Q,{ }_{N}^{2} \leq G / c^{4}$, equation (11.9) $)$ is equivalent to $\widetilde{F}_{3}>0$, where $\widetilde{F}_{3}(x) \stackrel{\text { def }}{=} F_{2}(x)-$ $\sqrt{F_{6}(x)}$. Note that $\widetilde{F}_{3}(0)=0$. We divide $\widetilde{F}_{3}(x)$ by $x^{2}$ and observe that the second term in the resulting expression is zero at $x=0$, while $\lim _{x \rightarrow 0} F_{2}(x) / x^{2}=6>0$. Thus, $\widetilde{F}_{3}>0$ in a vicinity of $x=0$, and, similarly to $F_{2}(x)$, tends to $+\infty$ as $x \rightarrow \infty$.

Since $\Gamma, \mathcal{M}=\Gamma,_{N} N, \mathcal{M}$, after using (5.16), we obtain:

$$
\frac{\Gamma \Gamma, \mathcal{M}}{2 E}=\frac{c^{4}}{G N_{0}} \frac{5}{6 x \sqrt{1+b x^{5 / 3}}},
$$

and so condition (8) becomes:

$$
F_{4}(x)>0, \quad \text { where } \quad F_{4}(x) \stackrel{\text { def }}{=} F_{1}(x)-\frac{5}{6 x \sqrt{1+b x^{5 / 3}}} F_{2}(x) .
$$

${ }^{5}$ We have $F_{2}(x)>\mu(x)-x /\left[(1+x)^{5} \sqrt{1+b x^{5 / 3}}\right] \stackrel{\text { def }}{=} \widetilde{F}_{2}$ for all $x>0$, and $\mathrm{d} \widetilde{F}_{2} / \mathrm{d} x>0$ for all $x>0$, so $F_{2}>\widetilde{F}_{2}>0$ for all $x>0$. 


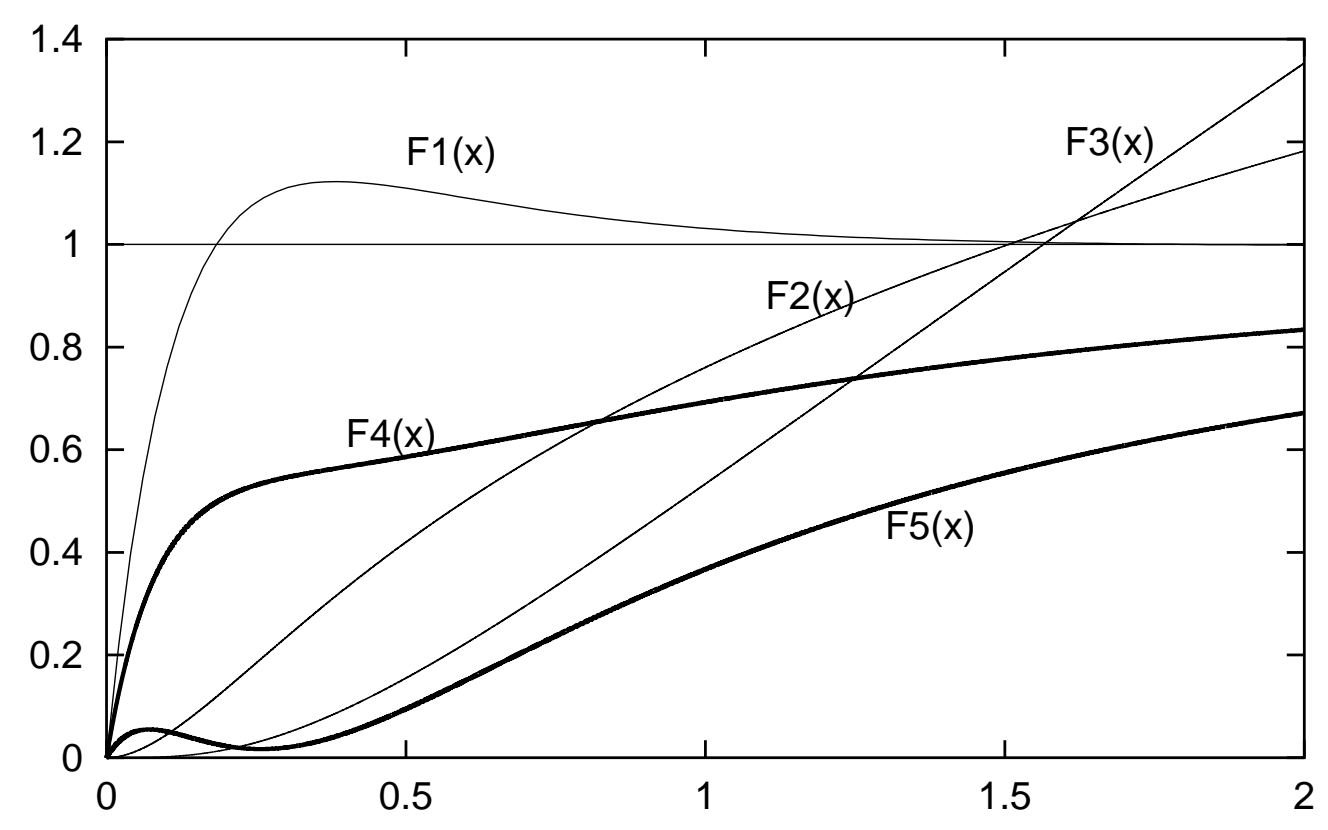

Figure 8: Graphs of the functions $F_{4}(x)$ and $F_{5}(x)$ with $b=2.5$. The graphs of $F_{1}(x), F_{2}(x)$ and $F_{3}(x)$ are shown for comparison and for scale. Both $F_{4}(x)$ and $F_{5}(x)$ asymptotically tend to 1 as $x \rightarrow \infty$ and both have vertical tangents at $x=0$.

We have $F_{4}(0)=0$. Knowing that $\lim _{x \rightarrow 0} F_{2} / x^{2}=6$, we easily calculate that $\mathrm{d} F_{4} /\left.\mathrm{d} x\right|_{x=0}=$ $7>0$. Thus $F_{4}(x)$ is increasing in a vicinity of $x=0$, i.e. $F_{4}(x)>0$ at least for some $x>0$. Also, $F_{4}(x) \underset{x \rightarrow \infty}{\longrightarrow} 1$. The graph of $F_{4}(x)$ with $b=2.5$ is shown in Fig. 8 ,

Finally, condition (9), i.e. eq. (10.2), can be written as

$$
F_{5}(x)>0, \quad \text { where } F_{5}(x) \stackrel{\text { def }}{=} F_{1}(x)-\frac{5}{6 x \sqrt{1+b x^{5 / 3}}}\left[F_{2}(x)+\sqrt{F_{3}(x)}\right],
$$

(see eq. (11.10) for the definition of $F_{6}(x)$ ). Since we have already verified that (11.12) holds, we can take the term with the square root in (11.13) to the r.h.s. in $F_{5}>0$ and then square both sides of the inequality. The result can be rewritten as:

$$
F_{1}\left(F_{1}-\frac{5}{3 x \sqrt{1+b x^{5 / 3}}} F_{2}\right)+\frac{25}{36 x^{2}\left(1+b x^{5 / 3}\right)} F_{6}>0 .
$$

We note that $\lim _{x \rightarrow 0} F_{1} / x=12, \lim _{x \rightarrow 0} F_{2} / x^{2}=6$ and $\lim _{x \rightarrow 0} F_{6} / x^{14 / 3}=8 b$. Then we divide (11.14) by $x^{2}$ and take the limit of the resulting expression at $x \rightarrow 0$. The result is $24>0$. Hence, (11.14) is obeyed in a vicinity of $x=0$, and, consequently, $F_{5}>0$ is obeyed in that vicinity, too. It is easy to verify that $F_{5}(x) \underset{x \rightarrow \infty}{\longrightarrow} 1$. The graph of $F_{5}$ with $b=2.5$ is shown in Fig. 8 .

The reason for choosing $b=2.5$ was this: the graph of $F_{5}$ is sensitive to the value of $b$. With decreasing $b$, the local minimum of $F_{5}$ becomes smaller, and for $b$ small enough (for example, $b=0.75) F_{5}<0$ around the minimum. With $b \geq 2.5$, the minimum is clearly positive.

Graphs do not constitute a definitive proof that a function is positive in the whole infinite range. Fine details can be hidden beyond the range of the figures or at a very small scale around the points where the functions approach zero. In those regions the 


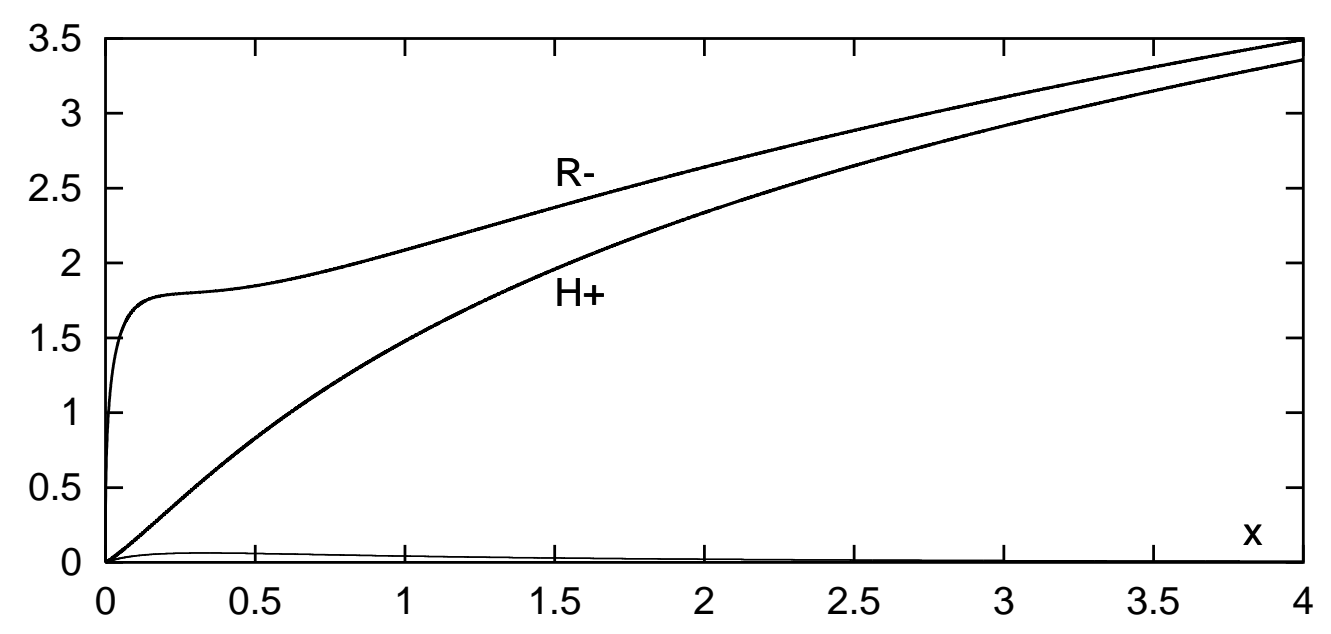

Figure 9: The maximal radius achieved in each cycle, $R_{-}$, and the radius of the outer $\mathrm{R}-\mathrm{N}$ horizon, $R_{H+}$, as functions of mass. The numbers on the axes are multiples of $G N_{0} / c^{4}$. At this scale, the radius of the inner horizon and the minimal radius seem to coincide with the $x$-axis, but see Fig. 10, As required by the equations of motion, we have $R_{-}>R_{H+}>R_{H_{-}}>R_{+}$for all masses. Note that $R_{-}$and $R_{H+}$ are increasing all the way.

functions can go below zero, while we cannot see it. However, we have verified by exact methods that there exists a neighbourhood $U$ of $x=0$ in which the functions $F_{1}, \ldots, F_{5}$ are positive for $x>0$. Thus, even if some of the functions become negative outside $U$, we can cut away a finite ball of the charged dust with the radius $x_{0}$ smaller than the radius of $U$ and match the charged dust to the Reissner - Nordström solution at $x=x_{0}$. In this way, we will obtain a finite charged body of dust that can go through a minimal size and bounce without encountering any singularity.

It can be easily verified that in our example $\left(G / c^{4}\right) Q^{2}<\mathcal{M}^{2}$ for all $x>0$ provided $b<25.3$ (hint: $p,_{x}<\mu,_{x}$ is equivalent to $\left[(1+x)^{6} /(1-x)^{2}-1\right]^{3 / 2}>b^{3 / 2} x^{5 / 2}-$ see the inset in Fig. (7). Thus, if a finite sphere is cut out of this configuration and matched to the Reissner - Nordström solution, the exterior $\mathrm{R}-\mathrm{N}$ metric will have $e^{2}<m^{2}$, and horizons will exist in it 6 As is well-known from the studies of the Reissner-Nordström metric [6], the surface of a charged dust sphere matched to the $\mathrm{R}-\mathrm{N}$ metric moves according to the same equation as a charged particle moving radially in an $\mathrm{R}-\mathrm{N}$ spacetime. Consequently, the reversal of collapse to expansion cannot occur between the two $\mathrm{R}-\mathrm{N}$ horizons. Our example is consistent with this. Fig. 9 shows the radii of the outer horizon and of the maximal radius achieved by a given $\mathcal{M}$ shell as functions of $x=N / N_{0}$. At the scale of Fig. 9, the minimal radius and the radius of the inner horizon seem to coincide with each other and with the $x$-axis. Fig. 10] shows a closeup view of the two curves, and shows that indeed the inner horizon has a smaller radius everywhere, except at the maximum, where the two curves are tangent to each other. This is no numerical coincidence - the two curves must be tangent at every local extremum of $R_{+}$.

The limit of the period (in $s$ ) of oscillations in (8.8) at $x \rightarrow 0$ is infinite. Thus, the limiting period in the time coordinate will also be infinite. Fig. 11 shows the numerically

\footnotetext{
${ }^{6} \mathrm{But} G Q^{2} /\left(c^{4} \mathcal{M}^{2}\right) \rightarrow 1$ as $x \rightarrow 0$, so as the sphere becomes smaller, the outer $\mathrm{R}-\mathrm{N}$ metric tends to the critical one, $e^{2}=m^{2}$.
} 


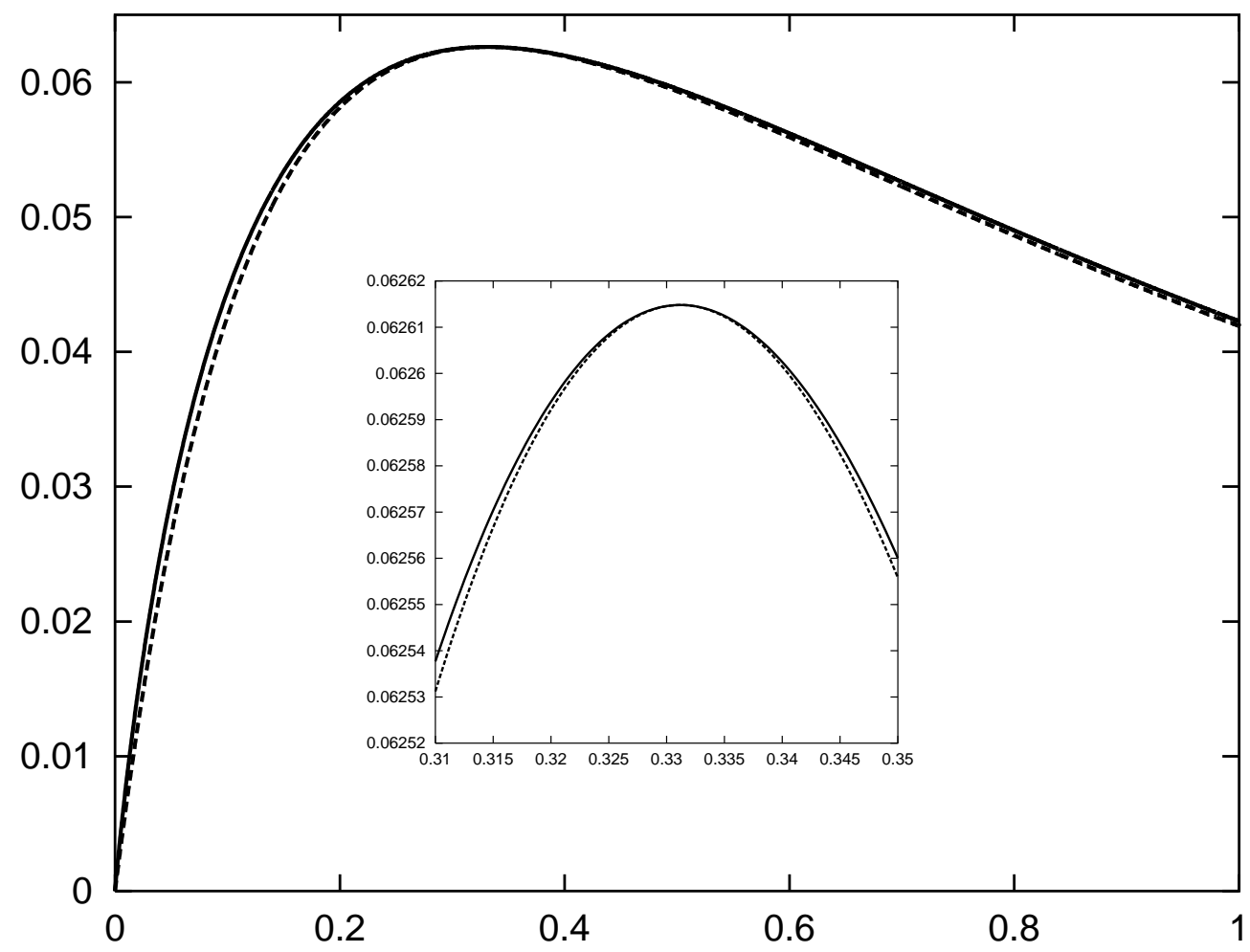

Figure 10: The radius of the inner horizon, $R_{H-}$ (upper curve), and the minimal radius, $R_{+}$(lower curve), as functions of mass. The numbers on the axes are multiples of $G N_{0} / c^{4}$. Both radii first increase with mass, but then decrease. The figure shows that $R_{+}<R_{H-}$ everywhere except at the maximum, where the two curves are tangent to each other. The inset is a closeup view of the neighbourhood of the maximum.

calculated period in $t$ as a function of $\mathcal{M}$, in the range $x \in[0,4)$ (for $x>4$ the calculation becomes progressively more difficult). As the figure shows, the period decreases with increasing $x$ in this whole range, so we will assume $x=4.0$ as a "practical" point of minimum of $T$. A real minimum must occur somewhere at $x>4.0$ because the period increases to $+\infty$ at both $x \rightarrow 0$ and $x \rightarrow+\infty$.

Finally, Fig. 12 shows a collection of curves $R(\mathcal{M}, t)$ corresponding to different values of $\mathcal{M}$, calculated numerically by solving the set (2.21) - (2.23). The configuration is timesymmetric with respect to the instant $t=0$. In agreement with Figs. 9, 10 and 11, the maximal and minimal radii achieved in each cycle are increasing functions of mass, while the period is a decreasing function 7

Figs. 12 and 11 are drawn on the basis of numerical solutions of the set (2.22) $-(2.23)$. As (2.22) shows, for small masses, where $Q \approx 0, C$ is nearly constant, i.e. nearly zero (because, as mentioned earlier, $t$ can be chosen so that $C=0$ at the center). Thus, the dependence of $R$ on $t$ for small values of $x$ should be very similar to the dependence of $R$ on $s$. Comparison of the corresponding graphs confirms this: the graphs of $R(\mathcal{M}, s)$ look identical to those of Fig. 12 and the period as a function of $s$ has a graph that looks identical to Fig. 11.

As predicted in Sec. 10, each mass shell avoids shell crossings throughout the first expansion phase after the time-symmetric bounce, but then experiences crossings after

\footnotetext{
${ }^{7}$ The range of masses in the figure is $x \in[0.01,0.1]$, where Figs. 9 , 10 and 11 show that the radii and the period should really behave the way they do in Fig. 12. For Figs. 11] and 12 the assumed value of $b$ is 0.001 .
} 


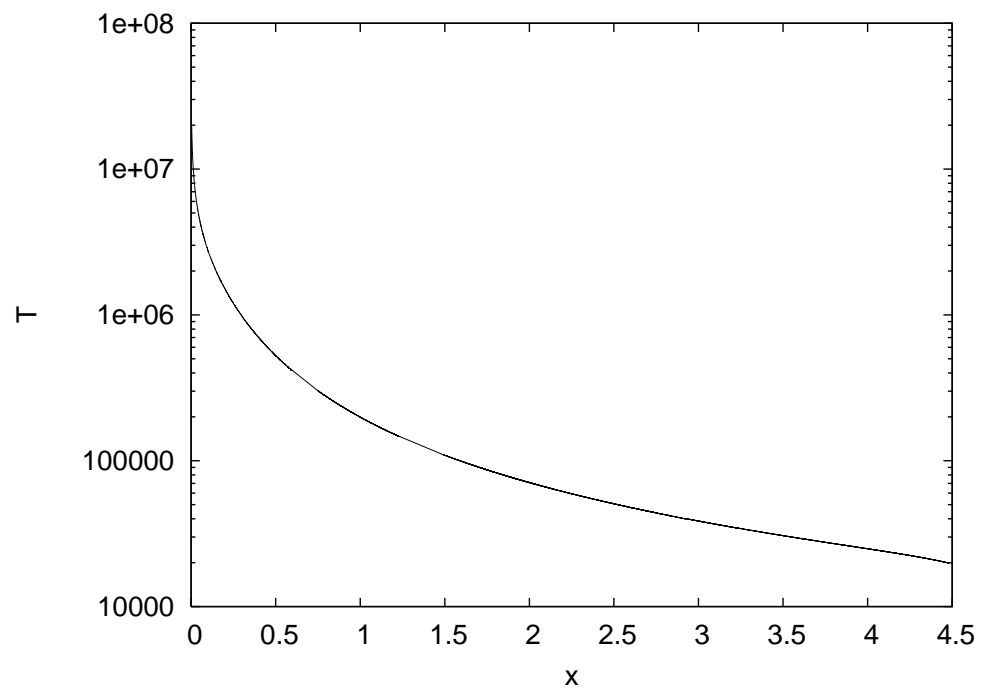

Figure 11: The period (in the time coordinate) as a function of $x=N / N_{0}$.

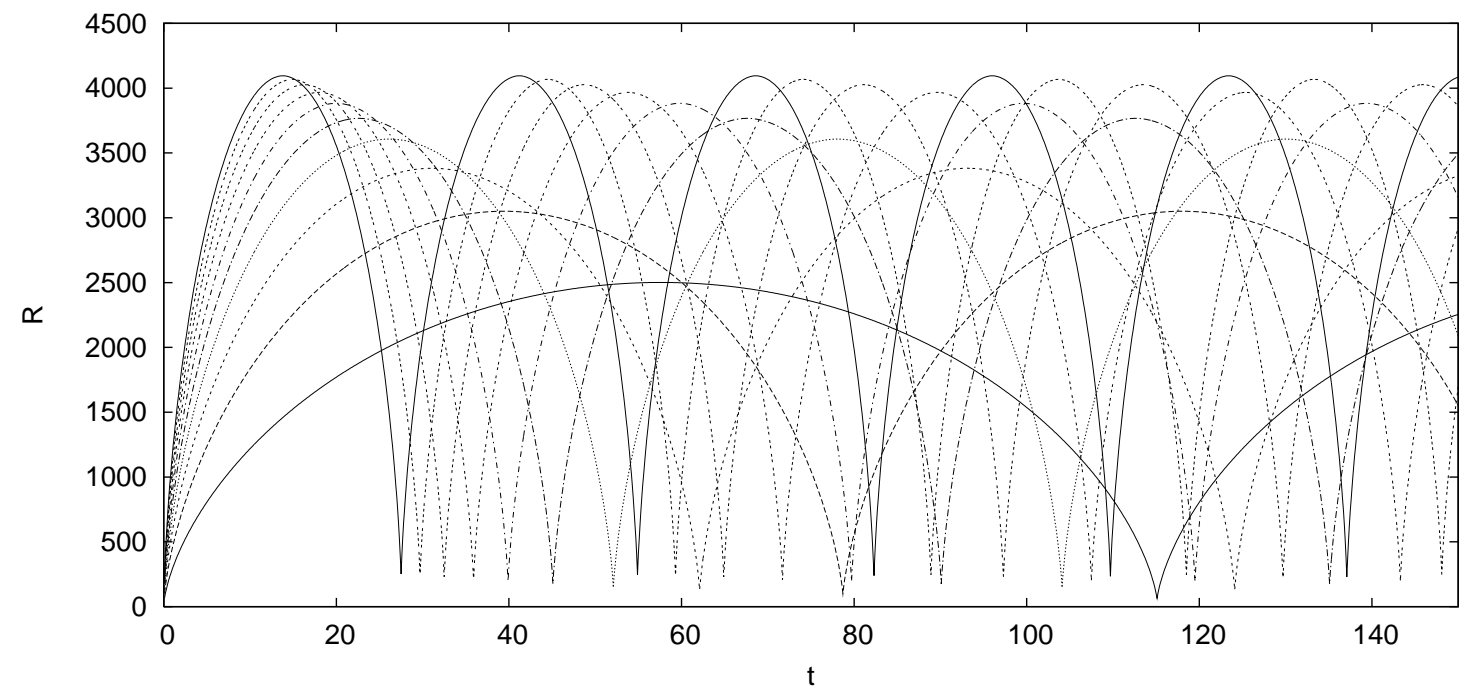

Figure 12: The curves $R(\mathcal{M}, t)$ corresponding to several values of $\mathcal{M}$. The mass increases uniformly from $x=0.01$ on the lowest curve to $x=0.1$ on the highest curve. The range of masses was chosen so as to make the figure readable; the real range of periods and of amplitudes can be very large. See more explanation in the text. The bounce is always smooth and at a nonzero value of $R$. (The figure suggests otherwise, but this is only an illusion created by the scale.) 
going through the maximal size. In order to avoid shell crossings in the whole volume for the whole expansion phase of the outermost shell, the radius of the dust ball cannot be too large. If it is very large, then the period of oscillations of the outermost shells will also become very large. Then, the time by which first shell crossings appear inside the ball will become a small fraction of the duration of the expansion phase of the outer surface, i.e. shell crossings will appear before the surface of the ball emerges from inside the outer horizon.

The evolution of our configuration is summarised in the Penrose diagram in Fig. 13. The diagram is written into the background of the Penrose diagram for the maximally extended Reissner-Nordström spacetime (thin lines). C is the center of symmetry, Sb is the surface of the charged ball, $\mathrm{S}_{\mathrm{RN}}$ is the Reissner-Nordström singularity. The interior of the body is encompassed by the lines C, E, Sb and B; no singularity occurs within this area. Lines $\mathrm{B}$ and $\mathrm{E}$ connect the points in spacetime where the shell crossings occur at different mass shells. N1 (N2) are the past- (future-) directed null geodesics emanating from the points in which the shell crossings reach the surface of the body (compare Fig. 12). The line Sb should be identified with the uppermost curve in Fig. 12, The top end of $\mathrm{Sb}$ is where the corresponding curve in Fig. 12 first crosses another curve, the middle point of $\mathrm{Sb}$ is at $t=0$ in Fig. 12,

It would be interesting to have an example of a configuration that can pulsate for ever, avoiding shell crossings in all of its collapse/expansion phases. However, our example does not obey (10.4) - substitution of our functions into (10.4) leads to a clearcut contradiction. Thus, it is impossible to impose on our example the condition that the period (in the proper time $s$ ) of pusations is independent of $\mathcal{M}$. Whether such a permanently pulsating singularity-free configuration exists at all is a problem to be investigated in the future.

We recall that our example was only meant to demonstrate that the inequalities in conditions $(1)-(9)$ are not mutually contradictory and allow a solution. Since it turned out that the set of models is not empty, more examples should exist, and now it is a challenge to explore other possibilities.

In the Friedmann limit (i.e. zero charge density, zero central charge and homogeneous mass density), a model with $E<0$ goes over to the $k>0$ Friedmann model, for which the most natural topology of spatial sections is that of $S^{3}$. Here we have a model that, if it were not matched to an $\mathrm{R}-\mathrm{N}$ spacetime, would have spatial sections of infinite volume (as attested by the fact that it can contain an infinite amount of rest mass). Lemaitre-Tolman models with such spatial topology are known and understood geometrically [15]. However, the limiting transition from such a model to the $k>0$ Friedmann model can be done only locally, i.e. in finite volumes, and involves a discontinuity in the arbitrary functions. Thus, it would be desirable to find a charged dust model with $E<0$ that would have complete spatial sections of finite volume. Curiously, it turned out to be much more difficult in this case to fulfil conditions $(7)-(9)$, and no example of such a model has been found. (The authors do not wish to imply that such an example does not exist, this is simply a problem to be solved.) 


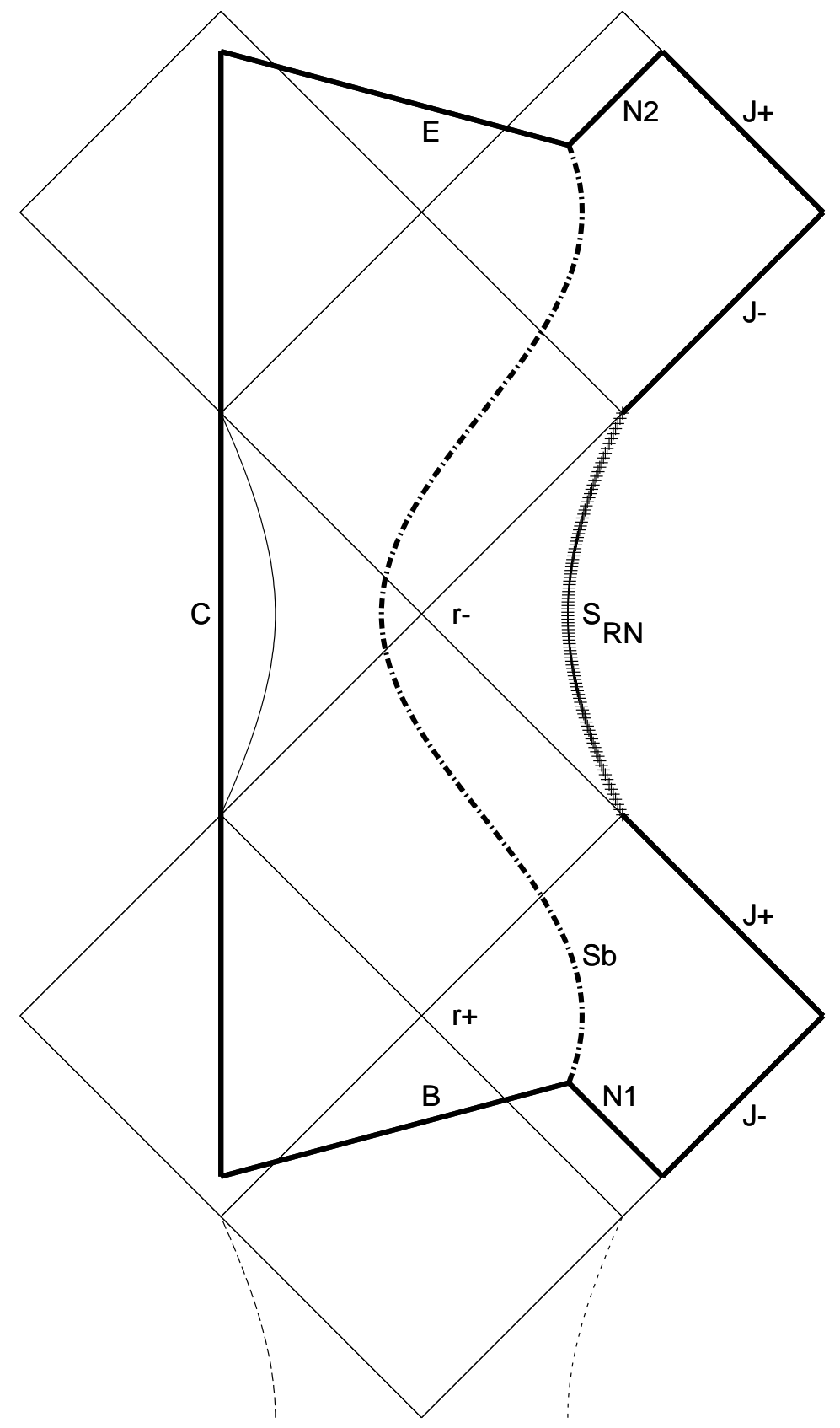

Figure 13: A schematic Penrose diagram for the configuration defined by eqs. (11.1) - (11.2) and (11.5). See explanation in the text. 


\section{Can such an object exist in the real Universe?}

There is a belief in the astronomical community that ordinary astronomical objects, such as stars and galaxies, have zero net charge. Quantitative estimates based on measurements and observations are, however, hard to come by. The best that can be found in the literature are theoretical considerations on how charges could be separated within the body of a star and what the maximal charge could be. Before we compare our results with those predictions, we shall first choose the most favourable parameters for our configuration.

It is clear that the smaller the net charge, the greater the chance that such an object might exist. For our object, the absolute value of the charge first increases from zero in the center to the maximum $\sqrt{G} N_{0} /\left(4 c^{2}\right)$ achieved at $x=1$, and then keeps decreasing all the way to zero as $x \rightarrow \infty$. It needs to be explained how such a charge could be concentrated toward the center. We shall not dwell on this mechanism because stars are obviously not composed of dust. In our case the concentration is a dynamical effect, achieved by fine-tuning of the initial positions and velocities.

As seen from Fig. 11, the period of oscillations decreases with mass, reaching a minimum at $x>4.0$. Then, Fig. 9 shows that at $x=4.0$, the maximal radius is slightly larger than the radius of the outer horizon. The period in $s$, calculated from (8.8), $2 \pi M /(-2 E)^{3 / 2}$, goes to $\infty$ both at $x \rightarrow 0$ and at $x \rightarrow \infty$, going through a minimum at $x \approx 1.0$. The period in $t$ should behave similarly, i.e. will start increasing at some $x=x_{m}$. We cannot take the radius of our object larger than $x_{m}$ because if it is large, then the period will be large, and shell crossings will appear inside the object before its surface emerges from the outer horizon. This would be unsatisfactory - we want the object to distinctly emerge from the horizon before any singularities destroy it. Thus, the largest total mass that we can assume corresponds to $x \approx 4.0$. At $x=4.0$, the charge is $|Q|=0.16 \sqrt{G} N_{0} / c^{2}$, while the active mass, in physical units 8 is $c^{2} \mathcal{M} / G=1.68 N_{0} / c^{2}$. The ratio of charge to mass is thus $G|Q| /\left(c^{2} \mathcal{M}\right)=0.095 \sqrt{G}$ in electrostatic units (in which the unit of charge is $\sqrt{\mathrm{g}}$ $\mathrm{cm}^{3 / 2} / \mathrm{s}$ ). This makes $0.82 \times 10^{-14}$ coulombs per gram. For the whole Earth, this ratio is $0.502 \times 10^{-25} \mathrm{C} / \mathrm{g}$ [16]. Compared to the charge of the Earth, the charge of our object is thus enormous. However, for a neutron star of 1 solar mass, the authors of Ref. [17] found that the total charge might be $10^{20} \mathrm{C}$, which makes $5.03 \times 10^{-14} \mathrm{C} / \mathrm{g}$ - distinctly more than in our object. Thus, the possibility to find a real object with charge and mass similar to our example is not outlandish 9 Only the mechanism of charge separation remains a problem.

We stress again that the functions defining our object were chosen rather accidentally, and lower ratios of charge to mass at the surface can possibly be achieved. But the limiting ratio of charge to mass at the center must be $\sqrt{G}$, which makes $8.616 \times 10^{-12} \mathrm{C} / \mathrm{g}$.

The period of oscillation, found from (8.8), is $T=2 \pi M /(-2 E)^{3 / 2}$, but this is expressed in geometrical units. In physical units, the period is $T / c$. Assuming that the surface of the object is at $x=4.0$, taking the corresponding mass to be $\mathcal{M}=1.68 G N_{0} / c^{4}$ and taking $b=2.5$, we calculate

$$
T / c=2.78 \times 10^{-38}\left(N_{0} / c^{2}\right),
$$

which, for solar mass $N_{0} / c^{2}=1.989 \times 10^{33} \mathrm{~g}$, gives $5.52 \times 10^{-5} \mathrm{~S}$ - the same order of

\footnotetext{
${ }^{8}$ What we have been calling 'active mass' $\mathcal{M}$ is in reality $\left(G / c^{2}\right) \times$ (mass).

${ }^{9}$ Note that we have not fixed the mass of our object - the constant $N_{0}$ is still arbitrary and can be made whatever we wish.
} 
magnitude as the time of collapse to the Schwarzschild singularity by a neutral star of one solar mass that has crossed the horizon. Needless to recall, for an observer at infinity in the $\mathrm{R}-\mathrm{N}$ spacetime, the time it takes a collapsing object to reach its horizon is infinite.

\section{Conclusions and possible further research}

We have shown that it is possible to set up such initial conditions for a charged dust sphere of a finite radius that its outer surface completes one full cycle of pulsation, while no singularity appears either at the surface or anywhere inside it. The sequence of events is this:

1. The initial instant is when the outer surface of the sphere is close to the maximum expansion. (When it is too late after the maximum, shell crossings in the interior will appear before the full cycle is completed; when it is too early before the maximum, shell crossings in the interior will exist already at the initial instant). Other surfaces of constant mass reach their maxima of expansion at (slightly) different instants of the time coordinate $t$. The outer surface at the initial instant is outside the outer Reissner-Nordström horizon.

2. During collapse, the outer surface plunges first through the outer $\mathrm{R}-\mathrm{N}$ horizon, then through the inner $\mathrm{R}-\mathrm{N}$ horizon, and bounces at a nonzero radius. No shell crossings appear anywhere inside the sphere during the collapse phase, and none of the constant-mass spheres collapse to zero radius, i.e. there are no singularities in this phase.

3. For all constant-mass shells, the bounce at minimal size is simultaneous in the timecoordinate t. This means that the evolution is time-symmetric with respect to this instant. This makes the problem technically simpler: if there were no singularities during collapse, there will be none during the next expansion phase.

4. The expanding outer surface goes out of the inner $\mathrm{R}-\mathrm{N}$ horizon, then out of the outer $\mathrm{R}-\mathrm{N}$ horizon and expands up to the maximal radius. As follows from the analysis of the maximally extended $\mathrm{R}-\mathrm{N}$ spacetime, after emerging from the outer $\mathrm{R}-\mathrm{N}$ horizon, the surface is in a different asymptotically flat sheet of the extended $\mathrm{R}-\mathrm{N}$ spacetime.

5. After the outer surface goes through the maximal radius, during the second collapse phase, shell crossing singularities appear inside the ball and its further evolution cannot be followed.

With the most favourable value of mass, the ratio of total charge to total mass of our dust ball is only slightly larger than the corresponding theoretically estimated maximum for charged neutron stars (see our Sec. 12 and Ref. [17]).

This is the description of our chosen example. However, other choices of the arbitrary functions are possible that might improve some of the characteristics of our model. For example, it would be desirable to avoid the matching to the $\mathrm{R}-\mathrm{N}$ spacetime, so that the dust distribution can extend over the whole space (infinite or closed) - then the solution could be investigated as a possible model of the Universe with a localised charged object in it. Another desirable generalisation would be to make all bounces time-symmetric, so that the model oscillates singularity-free for ever. We shall now briefly discuss these possibilities. Recall that one of the necessary conditions for the absence of singularities is $E<0$, and we shall assume this in the following.

Let us recall the necessary conditions for avoiding the $\mathrm{BB} / \mathrm{BC}$ and shell-crossing singularities. Two conditions are most important: 
1. The absolute value of the ratio of charge density to mass density at the center must be (in the units used here) $\sqrt{G} / c$ (see (2.20)). Otherwise, either shell crossings are inevitable or the ratio of charge density to mass density must be large throughout the volume to prevent $\mathrm{BC}$.

2. The limit of the period of oscillations at zero radius must not be zero, so that the pathological situation of Fig. [6 does not occur.

In addition to that, the following conditions must be obeyed:

3. The regularity conditions at the center of Sec. 6, which are the required values of various functions at $N=0$.

4. The 9 conditions listed in Sec. 10, most of which are inequalities to hold throughout a neighbourhood of the center. Of these, the functions in conditions (1) and (6-9) must have zero limits at the center.

All these conditions are difficult to fulfil because there are only two arbitrary functions in the model: ( $i$ ) the charge distribution $Q(N)$, and (ii) the energy distribution $E(N)$ (which can equivalently be defined by specifying $\Gamma(N)$ or $\mathcal{M}(N)$ ). Our choice here was the first $(Q, E)$ pair, found by trial and error, that obeys all the requirements, but only in a limited volume and in a limited time interval.

To avoid the artificially limited volume, one might try to match the model to a spacetime different from R-N. For example, it could be the Vickers model with the charge density becoming strictly zero at a certain distance from the center. Then, in the outer region, $Q,_{N}=0$, which is sufficient to prevent the $\mathrm{BB} / \mathrm{BC}$ singularity. Since the main difficulty in avoiding shell crossings was close to the center, and the $Q,{ }_{N}=0$ region would not extend to the center, chances are that shell crossings could be avoided as well. In the extreme case, the outer region could be the Lemaitre-Tolman spacetime. Since the L-T spacetime has strictly zero electric field, the function $Q(N)$ would have to be zero at the surface of the charged region. The zero total charge would be an advantage from the point of view of astronomy. We do not know if such a configuration exists.

To avoid the limited time interval, the bounce at minimal radius should be simultaneous in $t$ for all constant-mass shells. This means that the period of oscillations (measured by the time-coordinate $t$ ) would have to be independent of mass. Technically, this means that the integral of (9.1) from the minimal $R_{+}$to the maximal $R_{-}$(both given by (4.3)) should be independent of $\mathcal{M}$. We have not been able to tackle this condition.

The condition to avoid zero limit of the oscillation period at the center is, however, quite simple. It is reasonable to require that the function $C$ has a finite value at the center, where $N=0$ - without this assumption, finite intervals of the time coordinate $t$ would correspond to infinite intervals of the proper time $s$. If this is the case, then it is enough to choose such functions that the period in $s$ has a nonzero limit at $N=0$. From (8.8), the condition for this is that $M /(-2 E)^{3 / 2}$ has a nonzero limit at $N \rightarrow 0$. As noted at the very end of Sec. 10, the necessary condition for this is: the limit calculated in (6.4) is zero.

\section{Acknowledgements}

We thank Amos Ori for extended correspondence on several subjects concerning charged dust, in particular on the shell crossings and the existence of nonsingular solutions. The discussion helped to clarify a few points that have been left unexplained by previous 
publications.

\section{References}

[1] B. Datt, Z. Physik 108, 314 (1938); English translation, with historical comments: Gen. Rel. Grav. 31, 1615 (1999).

[2] V. A. Ruban, Pisma v Red. ZhETF 8, 669 (1968); English translation: Sov. Phys. JETP Lett. 8, 414 (1968); reprinted, with historical comments: Gen. Rel. Grav. 33, 363 (2001).

[3] V. A. Ruban, ZhETF 56, 1914 (1969); English translation: Sov. Phys. JETP 29, 1027 (1969); reprinted, with historical comments: Gen. Rel. Grav. 33, 375 (2001).

[4] V. A. Ruban, in: Tezisy dokladov 3-y Sovetskoy Gravitatsyonnoy Konferentsii [Theses of Lectures of the 3rd Soviet Conference on Gravitation]. Izdatel'stvo Erevanskogo Universiteta, Erevan (1972), p. 348.

[5] A. Krasiński, Inhomogeneous Cosmological Models. Cambridge University Press (1997).

[6] J. Plebański and A. Krasiński, An Introduction to General Relativity and Cosmology. Cambridge University Press, to appear in 2006.

[7] A. Ori, private communication.

[8] P. Noundjeu, Class. Quant. Grav. 22, 5365 (2005).

[9] P. A. Vickers, Ann. Inst. Poincarè A18, 137 (1973).

[10] I. S. Shikin, Commun. Math. Phys. 26, 24 (1972).

[11] A. Ori, Class. Q. Grav. 7, 985 (1990).

[12] A. Ori, Phys. Rev. D44, 2278 (1991).

[13] C. Hellaby and K. Lake, Astrophys. J. 290, 381 (1985) + erratum in Astrophys. J. 300, 461 (1985).

[14] A. Krasiński and C. Hellaby, Phys. Rev. D69, 023502 (2004).

[15] A. Krasiński and C. Hellaby, Phys. Rev. D69, 043502 (2004).

[16] W. Mizerski and W. Nowaczek, Tablice Fizyczno-Astronomiczne. Wydawnictwo Adamantan, Warszawa 1995, p. 69.

[17] S. Ray, W. Malheiro, J. P. S. Lemos and V. T. Zanchin, Braz. J. Phys. 34 no 1A, 310 (2004). 